New regression model with four regression structures and computational aspects Peer-reviewed author version

Ramires, Thiago G.; Ortega, Edwin M. M.; Cordeiro, Gauss M.; Paula, Gilberto A. \& HENS, Niel (2018) New regression model with four regression structures and computational aspects. In: COMMUNICATIONS IN STATISTICS-SIMULATION AND COMPUTATION, 47(7), p. 1940-1962.

DOI: $10.1080 / 03610918.2017 .1332212$

Handle: http://hdl.handle.net/1942/27427 


\section{Communications in Statistics - Simulation and Computation}

\section{New regression model with four regression structures and computational aspects}

\section{Thiago G. Ramires, Edwin M.M. Ortega, Gauss M. Cordeiro, Gilberto A. Paula \& Niel Hens}

To cite this article: Thiago G. Ramires, Edwin M.M. Ortega, Gauss M. Cordeiro, Gilberto A. Paula \& Niel Hens (2017): New regression model with four regression structures and computational aspects, Communications in Statistics - Simulation and Computation, DOI: 10.1080/03610918.2017.1332212

To link to this article: http://dx.doi.org/10.1080/03610918.2017.1332212

Accepted author version posted online: 26 May 2017.

Submit your article to this journal 7

View related articles

View Crossmark data 


\title{
New regression model with four regression structures and computational aspects
}

\author{
Thiago G. Ramires \\ Department of Exact Sciences, University of São Paulo, Brazil \\ Interuniversity Institute for Biostatistics and statistical Bioinformatics (I-Biostat), \\ University of Hasselt, Belgium; \\ Edwin M.M. Ortega \\ Department of Exact Sciences, University of São Paulo, Brazil \\ Gauss M. Cordeiro \\ Department of Statistics, Federal University of Pernambuco, Brazil \\ Gilberto A. Paula \\ Department of Statistics, Institute of Mathematics and Statistics, USP, Brazil \\ Niel Hens \\ Interuniversity Institute for Biostatistics and statistical Bioinformatics (I-Biostat), \\ University of Hasselt, Belgium; \\ Centre for Health Economic Research and Modelling Infectious Diseases, \\ Vaccine and Infectious Disease Institute, University of Antwerp, Belgium
}

\begin{abstract}
A new general class of exponentiated sinh Cauchy regression models for location, scale and shape parameters is introduced and studied. It may be applied to censored data and used more effectively in survival analysis when compared with the usual models. For censored data, we employ a frequentist analysis for the parameters of the proposed model. Further, for different parameter settings, sample sizes and censoring percentages, various simulations are performed. The extended regression model is very useful for the analysis of real data and could give more adequate fits than other special regression models.

Keywords: Exponentiated sinh Cauchy regression model; diagnostics analysis; GAMLSS; survival analysis.
\end{abstract}

\section{Introduction}

The Weibull, log-normal, log-logistic and Birnbaum-Saunders regression models are usually applied in science and engineering to model lifetime data for which linear functions of unknown parameters are 


\section{ACCEPTED MANUSCRIPT}

adapted to explain the phenomena under study. However, it is well-known that several phenomena are not always in agreement with the usual model due to lack of asymmetry, bimodality or the presence of heavily and lightly tailed distributions. In order to deal with this problem, some proposals have been made in literature with more flexible classes of distributions. We work with the exponentiated sinh Cauchy distribution because of its great flexility to fit asymmetric and bimodal data.

A large number of new distributions to extend well-known distributions and to provide flexibility in modeling data has being investigated in the last years. In this context, Gupta et al. (1998) pioneered a generalization of the standard exponential distribution called the exponentiated exponential (ExpE) distribution. The exponentiated class of distributions (Gupta and Kundu, 2001) has cumulative distribution function (cdf) given by

$$
F(t)=G(t)^{\tau}
$$

where $G(t)$ represents the baseline cdf and $\alpha>0$ denotes the shape parameter. By differentiating (1), the corresponding probability density function (pdf) becomes

$$
f(x)=\tau G(t)^{\tau-1} g(t)
$$

where $g(t)$ denotes the baseline pdf.

For modeling a lifetime $T>0$, Ramires et al. (2016) used the log-sinh Cauchy (LSC) distribution for the baseline in (2) by defining the four-parameter exponentiated log-sinh Cauchy (ELSC) distribution, whose pdf (for $t>0$ ) is given by

$$
f(t ; \mu, \sigma, \nu, \tau)=\frac{\tau \nu}{t \sigma \pi} \frac{\cosh \left(\frac{\log (t)-\mu}{\sigma}\right)}{\left[\nu^{2} \sinh ^{2}\left(\frac{\log (t)-\mu}{\sigma}\right)+1\right]}\left\{\frac{1}{2}+\frac{1}{\pi} \arctan \left[\nu \sinh \left(\frac{\log (t)-\mu}{\sigma}\right)\right]\right\}^{\tau-1},
$$

where $\mu \in \mathbb{R}$ and $\sigma>0$ are the location and scale parameters, respectively, $\nu>0$ is the symmetry parameter, which characterizes the bimodality of the distribution, and $\tau>0$ is the skewness parameter. The distribution of the $\operatorname{logarithm} Y=\log (T)$ is called the exponentiated sinh Cauchy (ESC) distribution, whose cdf (for $y \in \mathbb{R}$ ) is given by

$$
F(y ; \mu, \sigma, \nu, \tau)=\left\{\frac{1}{2}+\frac{1}{\pi} \arctan \left[\nu \sinh \left(\frac{y-\mu}{\sigma}\right)\right]\right\}^{\tau} .
$$

The pdf and survival function corresponding to (4) are given by

$$
f(y ; \mu, \sigma, \nu, \tau)=\frac{\tau \nu}{\sigma \pi} \frac{\cosh \left(\frac{y-\mu}{\sigma}\right)}{\left[\nu^{2} \sinh ^{2}\left(\frac{y-\mu}{\sigma}\right)+1\right]}\left\{\frac{1}{2}+\frac{1}{\pi} \arctan \left[\nu \sinh \left(\frac{y-\mu}{\sigma}\right)\right]\right\}^{\tau-1}
$$

and

$$
S(y ; \mu, \sigma, \nu, \tau)=\frac{(2 \pi)^{\tau}-\left\{\pi+2 \arctan \left[\nu \sinh \left(\frac{y-\mu}{\sigma}\right)\right]\right\}^{\tau}}{(2 \pi)^{\tau}},
$$

respectively. The ESC distribution (5) was first introduced by Cooray (2013) to modeling symmetric, right and left skewed and bimodal data sets. For $\tau=1$, the sinh Cauchy (SC) distribution is just a special case of (5). 


\section{ACCEPTED MANUSCRIPT}

In this paper, we propose a general class of regression models, where the mean, dispersion, asymmetry and bimodal parameters vary across observations through regression structures, assuming that the model errors follow the ESC distribution, which may be a useful alternative for modeling the four existing types of failure rate functions. The inferential component is carried out using the asymptotic distribution of the maximum likelihood estimators (MLEs). We also present methodologies to detect influential subjects with censored data and residual analysis for the proposed model. The script used to fit the ESC model, which is implemented in the R software environment ( $\mathrm{R}$ Core Team, 2015), is given in the Appendix.

The sections are organized as follows. In Section 2, we derive a power series for the quantile function (qf) and give explicit expressions for the moments. We propose an ESC regression model for modeling simultaneously the location, scale, bimodality and asymmetry parameters for censored data and discuss inferential issues in Section 3. Section 4 contains some Monte Carlo simultaneously on the finite sample behavior of the MLEs. In Section 5, we assess the behavior of the MLEs of the parameters in the ESC regression model when it is poorly specified. In Section 6, we discuss some diagnostic measures for three perturbation schemes, case-deletion and generalized leverage method. The residuals from a fitted model using the martingale residual and martingale-type residual are also presented in this section. Applications to two real data sets are addressed in Section 7 to illustrate the flexibility of the proposed class of regression models for censored and uncensored data. Finally, Section 8 offers some conclusions.

\section{Properties of the standardized ESC distribution}

In this section, we study some properties of the standard ESC random variable defined by $Z=$ $(Y-\mu) / \sigma$. The density function of $Z$ (for $z \in \mathbb{R}$ ) reduces to

$$
f(z ; \nu, \tau)=\tau g_{\mathcal{S C}}(z) G_{\mathcal{S C}}(z)^{\tau-1}=\frac{\tau \nu}{\pi} \frac{\cosh (z)}{\nu^{2} \sinh ^{2}(z)+1}\left\{\frac{1}{2}+\frac{1}{\pi} \arctan [\nu \sinh (z)]\right\}^{\tau-1}
$$

where $G_{\mathcal{S C}}(z)$ and $g_{\mathcal{S C}}(z)$ denote the cdf and pdf of standard SC distribution given by

$$
G_{\mathcal{S C}}(z)=\left\{\frac{1}{2}+\frac{1}{\pi} \arctan [\nu \sinh (z)]\right\} \quad \text { and } \quad g_{\mathcal{S C}}(z)=\frac{\nu}{\pi} \frac{\cosh (z)}{\nu^{2} \sinh ^{2}(z)+1},
$$

respectively.

Plots of the density function (7) for selected parameter values are displayed in Figure 1. Equation (7) for the standardized ESC distribution will be used in Section 3.1 to specify the error distribution of the proposed regression model.

\subsection{Expansion of the quantile function}

Inverting $F(y)=u$ in (4) gives the qf of $Y$

$$
Y=Q_{Y}(u)=\mu+\sigma \operatorname{arcsinh}\left\{\frac{1}{\nu} \tan \left[\pi\left(u^{1 / \tau}-0.5\right)\right]\right\} .
$$




\section{ACCEPTED MANUSCRIPT}

(a)

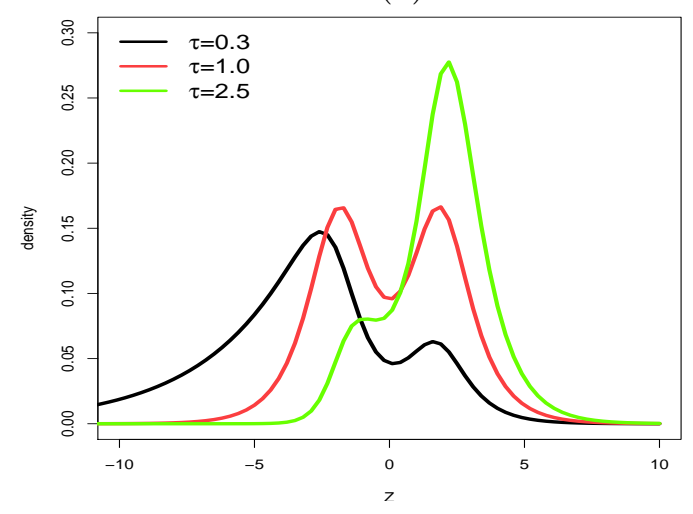

(b)

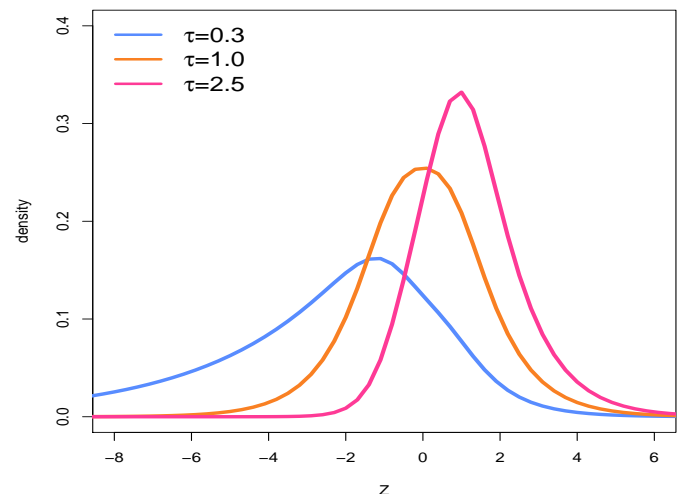

Figure 1: Plots of the density function (7) for some values of $\tau$ : (a) $\nu=0.3$; (b) $\nu=0.8$.

The qf $Q_{Z}(u)$ of $Z$, which has the standardized ESC density function (7), can be obtained from (9) with $\mu=0$ and $\sigma=1$. The qf of the standardized SC distribution, say $Q_{S C}(u)$, also follows (9) with $\mu=0$ and $\sigma=\tau=1$ and it will be used to demonstrate some properties of $Z$ in the following sections.

We can use (9) for simulating ESC or standardized ESC random variables by setting $u$ as a uniform random variable in the interval $(0,1)$. The qf is widely used to determine some mathematical properties like moments, generating function, Galton's skewness and Moors's kurtosis. Recently, Ortega et al. (2016) used the qf to demonstrate some properties of the log-odds Birnbaum-Saunders model and Cordeiro et al. (2016) presented those for the generalized odd half-Cauchy family.

Next, we derive a power series for the qf of $Z$. Expanding (9) in Mathematica in a power series, considering $\mu=0$ and $\sigma=1$, we have

$$
Q_{Z}(u)=\sum_{k=0}^{\infty} c_{k}\left(u^{1 / \tau}-0.5\right)^{2 k+1},
$$

where $c_{k}=\frac{b_{k}}{(2 k+1) !}\left(\frac{\pi}{\nu}\right)^{2 k+1}$ and $b_{0}=1, b_{1}=2 \nu^{2}-1, b_{2}=16 \nu^{4}-20 \nu^{2}+9, b_{3}=272 \nu^{6}-616 \nu^{4}+$ $630 \nu^{2}-225, b_{4}=7936 \nu^{8}-28160 \nu^{6}+48384 \nu^{4}-37800 \nu^{2}+11025, \ldots$

By expanding the binomial term, the last equation reduces to

$$
Q_{Z}(u)=\sum_{k=0}^{\infty} \sum_{j=0}^{\infty} \frac{(-1)^{2 k+1-j} u^{j / \tau}}{2^{2 k+1-j}}\left(\begin{array}{c}
2 k+1 \\
j
\end{array}\right) c_{k} .
$$

Finally, changing $\sum_{k=0}^{\infty} \sum_{j=0}^{\infty}$ by $\sum_{j=0}^{\infty} \sum_{k=j}^{\infty}$, we obtain

$$
Q_{Z}(u)=\sum_{j=0}^{\infty} p_{j} u^{j / \tau}
$$

where the coefficients

$$
p_{j}=\sum_{k=j}^{\infty}(-0.5)^{2 k+1-j}\left(\begin{array}{c}
2 k+1 \\
j
\end{array}\right) c_{k}
$$

can be determined using e.g. Mathematica, Maple, R and Sage. 


\section{ACCEPTED MANUSCRIPT}

\subsection{Moments}

Let $\mu_{s}^{\prime}=E\left(Z^{s}\right)$ be the $s$ th ordinary moment of $Z$ with pdf (7). We have

$$
\mu_{s}^{\prime}=\tau \int_{-\infty}^{\infty} z^{s} g_{\mathcal{S C}}(z) G_{\mathcal{S C}}(z)^{\tau-1} d z=\tau \int_{0}^{1} Q_{S C}(u)^{s} u^{\tau-1} d u
$$

Replacing $Q_{S C}(u)$ (eq. (10) when $\tau=1$ ) in the last equation, we obtain

$$
\mu_{n}^{\prime}=\tau \int_{0}^{1}\left(\sum_{j=0}^{\infty} p_{j} u^{j}\right)^{s} u^{\tau-1} d u .
$$

Henceforth, we use an equation by Gradshteyn and Ryzhik (2007) for a power series raised to a positive integer $n$

$$
\left(\sum_{i=0}^{\infty} a_{i} u^{i}\right)^{n}=\sum_{i=0}^{\infty} b_{n, i} u^{i}
$$

where the coefficients $b_{n, i}$ (for $i=1,2, \ldots$ ) are easily determined from the recurrence equation

$$
b_{n, i}=\left(i a_{0}\right)^{-1} \sum_{m=1}^{i}[m(n+1)-i] a_{m} b_{n, i-m},
$$

and $b_{n, 0}=a_{0}^{n}$. The coefficient $b_{n, i}$ can be determined numerically from the quantities $a_{0}, \ldots, a_{i}$.

Based on equation (13), equation (12) can be rewritten as

$$
\mu_{n}^{\prime}=\tau \sum_{j=0}^{\infty} e_{s, j} \int_{0}^{1} u^{j+\tau-1} d u=\sum_{j=0}^{\infty} \frac{\tau}{\tau+j} e_{s, j},
$$

where $e_{s, j}=\frac{1}{j p_{0}} \sum_{m=1}^{j}[m(s+1)-j] p_{m} e_{s, j-m}, e_{s, 0}=p_{0}^{s}$, and $p_{0}$ and $p_{m}$ are obtained by (11).

The skewness and kurtosis measures can be calculated from the ordinary moments using wellknown relationships. Plots of the skewness and kurtosis of $Z$ are displayed in Figures 2 and 3 for selected values of $\tau$ as functions of $\nu$ and for selected values of $\nu$ as functions of $\tau$, respectively.

\section{The ESC regression model}

In many practical applications, the lifetimes are affected by explanatory variables such as blood pressure, weight, cholesterol level and many others. Parametric models for estimating univariate survival functions and for the censored data regression problems are widely used. When the parametric models provide good fits to lifetime data, they tend to provide more precise estimates for the quantities of interest because these estimates are based on fewer parameters. Recently, several regression models have been proposed in literature by considering the class of location models. For example, Hashimoto et al. (2012) proposed the log-Burr XII regression model for grouped survival data, Ortega et al. (2013) presented the log-beta Weibull regression model for predicting recurrence of prostate cancer, Ortega et al. (2015) studied a power series beta Weibull regression model for predicting breast carcinoma, etc. 


\section{ACCEPTED MANUSCRIPT}

(a)

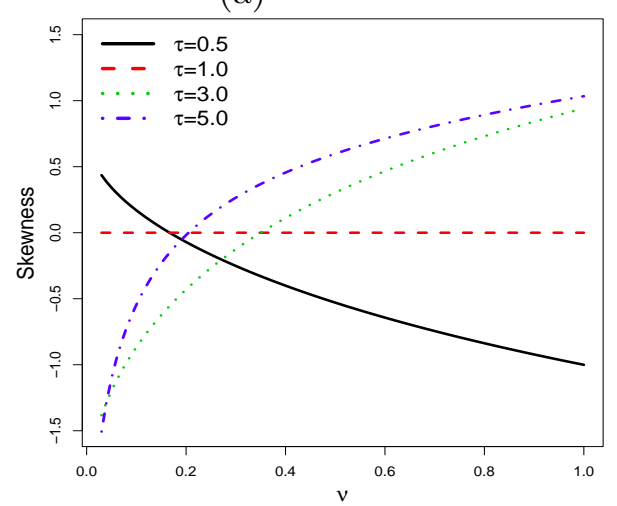

(b)

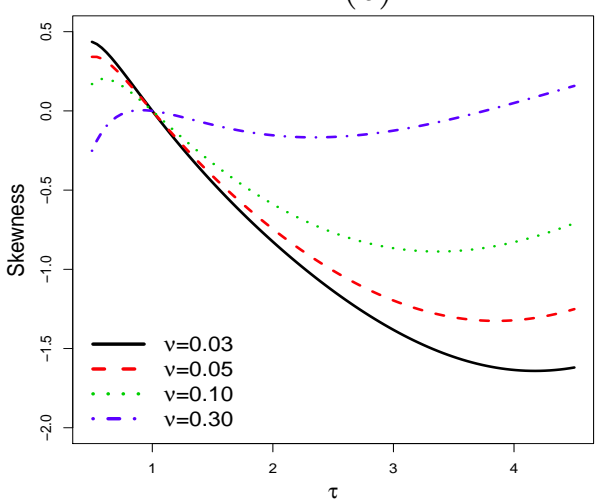

Figure 2: Skewness of the ESC distribution: (a) Function of $\nu$ for some values of $\tau$. (b) Function of $\tau$ for some values of $\nu$.

(a)

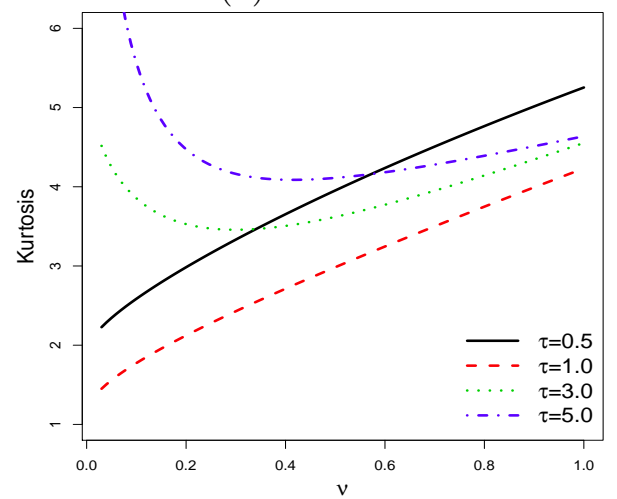

(b)

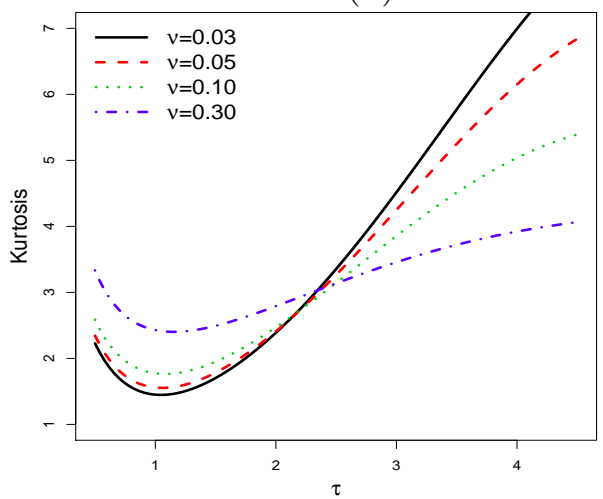

Figure 3: Kurtosis of the ESC distribution: (a) Function of $\nu$ for some values of $\tau$. (b) Function of $\tau$ for some values of $\nu$.

A disadvantage of the class of location model is that the variance, skewness, bimodality, kurtosis and other parameters can not be modelled explicitly in terms of explanatory variables but implicitly through their dependence on the location parameter. As an alternative, the generalized additive models for location, scale and shape (GAMLSS) (Rigby and Stasinopouls, 2005), where the systematic part of the model is expanded to allow not only the location but all the parameters of the conditional distribution of $Y$ to be modelled as parametric functions of explanatory variables, become widely used. In this sense, we introduce the ESC regression model following the GAMLSS set-up.

\subsection{Definition}

Let $\boldsymbol{\theta}^{T}=(\mu, \sigma, \nu, \tau)$ denote the vector of parameters of the pdf (5). We consider that independent observations $y_{i}$ conditional on $\boldsymbol{\theta}_{i}$ (for $\left.i=1, \ldots, n\right)$, with pdf $f\left(y_{i} ; \boldsymbol{\theta}_{i}\right)$, where $\boldsymbol{\theta}_{i}^{T}=\left(\mu_{i}, \sigma_{i}, \nu_{i}, \tau_{i}\right)$ is a parameter vector related to the response variable.

Based on the ELSC distribution, we propose a linear regression model linking the response variable 


\section{ACCEPTED MANUSCRIPT}

$y_{i}$ and the explanatory variable by

$$
y_{i}=\mu_{i}+\sigma_{i} z_{i}, \quad i=1, \ldots, n,
$$

where the random error $z_{i}$ follows the density function $f\left(z_{i} ; \nu_{i}, \tau_{i}\right)$ given by $(7)$ and $Z_{i}=\left(Y_{i}-\mu_{i}\right) / \sigma_{i}$. We define the parameter vector $\boldsymbol{\theta}$ using appropriate link functions as

$$
\boldsymbol{\theta}=\left[\begin{array}{c}
\boldsymbol{\mu} \\
\boldsymbol{\sigma} \\
\boldsymbol{\nu} \\
\boldsymbol{\tau}
\end{array}\right]=\left[\begin{array}{c}
g_{1}\left(\mathbf{X}_{1} \boldsymbol{\beta}_{1}\right) \\
g_{2}\left(\mathbf{X}_{2} \boldsymbol{\beta}_{2}\right) \\
g_{3}\left(\mathbf{X}_{3} \boldsymbol{\beta}_{3}\right) \\
g_{4}\left(\mathbf{X}_{4} \boldsymbol{\beta}_{4}\right)
\end{array}\right] \text { or } \theta_{i}=\left[\begin{array}{c}
\mu_{i} \\
\sigma_{i} \\
\nu_{i} \\
\tau_{i}
\end{array}\right]=\left[\begin{array}{c}
g_{1}\left(\beta_{01}+x_{1}[i, 2] \beta_{11}+\ldots+x_{1}\left[i, p_{1}+1\right] \beta_{p_{1} 1}\right) \\
g_{2}\left(\beta_{02}+x_{2}[i, 2] \beta_{12}+\ldots+x_{2}\left[i, p_{2}+1\right] \beta_{p_{2} 2}\right) \\
g_{3}\left(\beta_{03}+x_{3}[i, 2] \beta_{13}+\ldots+x_{3}\left[i, p_{3}+1\right] \beta_{p_{3} 3}\right) \\
g_{4}\left(\beta_{04}+x_{4}[i, 2] \beta_{14}+\ldots+x_{4}\left[i, p_{4}+1\right] \beta_{p_{4} 4}\right)
\end{array}\right],
$$

where $p_{k}$ denotes the number of explanatory variables related to the $k$ th parameter, $g_{1}(\cdot)$ is an injective and twice continuously differentiable function, $g_{k}(\cdot)$ (for $k=2,3,4$,) is a known positive continuously differentiable function containing values of the explanatory variables, $\boldsymbol{\beta}_{k}=\left(\beta_{0 k}, \beta_{1 k}, \ldots, \beta_{p_{k} k}\right)^{T}$ is a parameter vector of length $\left(p_{k}+1\right), \mathbf{X}_{k}$ is a known model matrix of order $n \times\left(p_{k}+1\right)$ and $x_{k}\left[i, p_{k}\right]$ are the elements of the matrix $\mathbf{X}_{k}$. The total number of parameters to be estimated is given by $p=p_{1}+p_{2}+p_{3}+p_{4}+4$. Note that we assume that four parameters $\mu_{i}, \sigma_{i}, \nu_{i}$ and $\tau_{i}$ vary across observations through regression structures. For the following sections, we shall consider the identity link function for $g_{1}(\cdot)$ and the logarithmic link function for $g_{k}(\cdot)$ (for $k=2,3,4$, ).

The sinh Cauchy (SC) regression model is obtained as a special case of (15) when $\tau_{i}=1$. The class of location is obtained when $p_{2}=p_{3}=p_{4}=0$. For $p_{3}=p_{4}=0, p_{1} \neq 0$ and $p_{2} \neq 0$, we also obtain the regression model with heteroscedastic errors, which can be used as an alternative to transformation of the response variable. However, the choice of parameters to be modeled by explanatory variables will depend on the data set.

\subsection{Estimation}

Consider a sample of $n$-independent observations, where each random response is defined by $y_{i}=$ $\min \left[\log \left(t_{i}\right), \log \left(c_{i}\right)\right]$. We assume non-informative censoring and that the observed lifetimes and censoring times are independent. Let $\mathrm{F}$ and $\mathrm{C}$ be the sets of individuals for which $y_{i}$ is the log-lifetime or logcensoring, respectively. The total log-likelihood function for the model parameters $\boldsymbol{\theta}=(\boldsymbol{\mu}, \boldsymbol{\sigma}, \boldsymbol{\nu}, \boldsymbol{\tau})^{T}$ from model (15) is given by $l(\boldsymbol{\theta})=\sum_{i \in F} \log f\left(y_{i} ; \boldsymbol{\theta}_{i}\right)+\sum_{i \in C} \log S\left(y_{i} ; \boldsymbol{\theta}_{i}\right)$, where $f\left(y_{i} ; \boldsymbol{\theta}_{i}\right)$ is the density function in (5) and $S\left(y_{i} ; \boldsymbol{\theta}_{i}\right)$ is the survival function in (6). The log-likelihood function for $\boldsymbol{\theta}$ reduces to

$$
\begin{aligned}
l(\boldsymbol{\theta})= & -\sum_{i \in F} \log \left[1+\nu_{i}^{2} \sinh ^{2}\left(z_{i}\right)\right]+\sum_{i \in F} \log \cosh \left(z_{i}\right)+\sum_{i \in F}\left(\tau_{i}-1\right) \log \left\{\frac{1}{2}+\frac{1}{\pi} \arctan \left[\nu_{i} \sinh \left(z_{i}\right)\right]\right\} \\
& +\sum_{i \in F} \log \left(\tau_{i} \nu_{i}\right)-\sum_{i \in F} \log \left(\sigma_{i} \pi\right)+\sum_{i \in C} \log \left(1-\left\{\frac{1}{2}+\frac{1}{\pi} \arctan \left[\nu_{i} \sinh \left(z_{i}\right)\right]\right\}^{\tau_{i}}\right) .
\end{aligned}
$$

The MLE $\widehat{\boldsymbol{\theta}}$ of the vector $\boldsymbol{\theta}^{T}=(\mu, \sigma, \nu, \tau)$ of unknown parameters can be evaluated by maximizing the log-likelihood (17) numerically in the GAMLSS package of the R software. The advantage of using this package is that we can adopt many maximization methods, which will depend only on the current fitted model. When there are no explanatory variables or censored observations, we can use 


\section{ACCEPTED MANUSCRIPT}

the gamlssML function for fitting (17) using a non-linear maximization algorithm. In the presence of censored observations, the additional package gamlss.cens is required to determine numerically the observed information of the likelihood function referring to the censored observations. The maximization procedures used in the presence of censored data are the generalizations of the Rigby and Stasinopoulos (RS) and Cole and Green (CG) algorithms. All methods and algorithms are described by Rigby and Stasinopouls (2005) and Stasinopoulos and Rigby (2007) and available in the GAMLSS package. The RS algorithm requires the first order derivatives of the logarithm of the density function (5) given in the above equations, and the second order derivatives. The RS method, different from the CG algorithm, does not use the cross derivatives, and thus it is faster for larger data sets.

An important consideration in the statistical analysis in regression models is the assumption that all observations have equal variances. The non-compliance with this assumption affects the efficiency of the estimates of the parameters. In particular, we now consider the test of homogeneity of variances for the ESC regression model based on the asymptotic distribution of the parameters. Under standard regularity conditions, the asymptotic distribution of $(\widehat{\boldsymbol{\theta}}-\boldsymbol{\theta})$ is $N_{p}\left(\mathbf{0}, I(\boldsymbol{\theta})^{-1}\right)$, where $I(\boldsymbol{\theta})$ is the expected information matrix. The multivariate normal $N_{p}\left(\mathbf{0}, \ddot{\mathbf{L}}(\widehat{\boldsymbol{\theta}})^{-1}\right)$ distribution can be used to construct approximate confidence intervals for the individual parameters, where $\ddot{\mathbf{L}}(\widehat{\boldsymbol{\theta}})$ is the observed information matrix. Following (16), we generalize the scale parameter $\sigma$ as $\boldsymbol{\sigma}=g_{2}\left(\mathbf{X}_{2} \boldsymbol{\beta}_{2}\right)$, where $\mathbf{X}_{i 2}$ is a matrix of explanatory variable values. For example, consider a matrix $\mathbf{X}_{2}(n \times 2)$ with the first column of ones corresponding to $\beta_{02}$, and the second column with the values of $x_{1}$ corresponding to $\beta_{12}$. We can test the homogeneity of variances between the levels (or ranges) of $x_{1}$ by testing the hypotheses $\mathcal{H}_{0}: \beta_{12}=0$ against $\mathcal{H}_{a}: \beta_{12} \neq 0$, where the Wald statistic is given by $T=\hat{\beta}_{12} / \sqrt{\ddot{\mathbf{L}}(\widehat{\boldsymbol{\theta}})_{\beta_{12}}^{-1}} \sim t_{(n-p-1)}$, and $\ddot{\mathbf{L}}(\widehat{\boldsymbol{\theta}})_{\beta_{12}}^{-1}$ is the $\left(p_{1}+2, p_{1}+2\right)$ element of the observed information matrix. Analogously, we can provide the same tests of hypotheses for the parameters $\boldsymbol{\mu}, \boldsymbol{\nu}$ and $\boldsymbol{\tau}$.

\section{Simulation Study}

We conduct two Monte Carlo simulation studies to assess the finite sample behavior of the MLEs of the parameters for different sample sizes " $n$ " and censoring percentages " $k$ ". In the first simulation, we consider the location model in (15), where $\mu_{i}=\beta_{01}+\beta_{11} x_{i}, \sigma_{i}=\sigma, \nu_{i}=\nu$ and $\tau_{i}=\tau$. In the second simulation, we consider the GAMLSS model in (15) by modeling the parameters using the explanatory variable $x_{i}$, namely: $\mu_{i}=\beta_{01}+\beta_{11} x_{i}, \sigma_{i}=\exp \left(\beta_{02}+\beta_{12} x_{i}\right), \nu_{i}=\exp \left(\beta_{03}+\beta_{13} x_{i}\right)$ and $\tau_{i}=\exp \left(\beta_{04}+\beta_{14} x_{i}\right)$.

In the two simulations, the sample sizes are generated by taking $n=50$ and 100 . The log-lifetimes denoted by $\log \left(T_{1}\right), \ldots, \log \left(T_{n}\right)$ are generated from the ESC distribution using the qf $(9)$, where the parameter vectors were fixed and evaluated using the explanatory variable $x_{i}$ generated from a uniform $(0,1)$ distribution. The censoring times, denoted by $C_{1}, \ldots, C_{n}$, are randomly generated for censoring percentages $\kappa=0.0,0.1$ and 0.3 , respectively.

The lifetimes considered in each fit are evaluated as $\min \left[\log \left(C_{i}\right), \log \left(T_{i}\right)\right]$. For each configuration of $n$ and $\kappa$, all results are obtained from 2,000 Monte Carlo replications and the simulations are carried out using the $\mathrm{R}$ programming language. For each replication, a random sample of size $n$ is drawn from the ESC regression model (15) for survival censored data and the optim algorithm is used for 


\section{ACCEPTED MANUSCRIPT}

maximizing the total log-likelihood function (17).

\subsection{Location simulation}

For the location model, the true parameter values used in the data-generating process are $\mu_{i}=1+3 x_{i}$, $\sigma=3, \nu=0.2$ and $\tau=2$. For each fit, the average estimates (AEs), biases and means squared errors (MSEs) are evaluated. The results are given in Table 1.

Table 1: The AEs, biases and MSEs based on 2,000 simulations for the location ESC regression model when $\beta_{01}=1, \beta_{11}=3, \sigma=3, \nu=0.2$ and $\tau=2$, for $n=50$ and 100 for censoring percentages $\kappa=0.0$, 0.1 and 0.3 .

\begin{tabular}{|c|c|c|c|c|c|c|c|c|}
\hline \multirow[b]{2}{*}{$\kappa$} & \multirow[b]{2}{*}{ Parameter } & \multicolumn{3}{|c|}{$n=50$} & \multirow[b]{2}{*}{ Parameter } & \multicolumn{3}{|c|}{$n=100$} \\
\hline & & $\mathrm{AE}$ & Bias & MSE & & $\mathrm{AE}$ & Bias & MSE \\
\hline \multirow[t]{5}{*}{0.0} & $\beta_{0}$ & 1.326 & 0.326 & 2.920 & $\beta_{0}$ & 1.185 & 0.185 & 1.033 \\
\hline & $\beta_{1}$ & 2.978 & -0.022 & 5.968 & $\beta_{1}$ & 3.044 & 0.044 & 2.117 \\
\hline & $\sigma$ & 2.628 & -0.372 & 0.280 & $\sigma$ & 2.704 & -0.296 & 0.152 \\
\hline & $\nu$ & 0.164 & -0.036 & 0.007 & $\nu$ & 0.171 & -0.029 & 0.003 \\
\hline & $\tau$ & 2.053 & 0.053 & 0.200 & $\tau$ & 2.063 & 0.063 & 0.102 \\
\hline \multirow[t]{5}{*}{0.1} & $\beta_{0}$ & 1.324 & 0.324 & 3.365 & $\beta_{0}$ & 1.039 & 0.039 & 1.248 \\
\hline & $\beta_{1}$ & 3.036 & 0.036 & 6.402 & $\beta_{1}$ & 3.414 & 0.414 & 3.029 \\
\hline & $\sigma$ & 2.732 & -0.268 & 0.222 & $\sigma$ & 2.817 & -0.183 & 0.123 \\
\hline & $\nu$ & 0.169 & -0.031 & 0.006 & $\nu$ & 0.174 & -0.026 & 0.003 \\
\hline & $\tau$ & 2.187 & 0.187 & 0.269 & $\tau$ & 2.188 & 0.188 & 0.143 \\
\hline \multirow[t]{5}{*}{0.3} & $\beta_{0}$ & 2.511 & 1.511 & 7.315 & $\beta_{0}$ & 0.986 & -0.014 & 1.564 \\
\hline & $\beta_{1}$ & 1.111 & -1.889 & 12.032 & $\beta_{1}$ & 3.450 & 0.450 & 3.121 \\
\hline & $\sigma$ & 3.024 & 0.024 & 0.332 & $\sigma$ & 3.142 & 0.142 & 0.185 \\
\hline & $\nu$ & 0.189 & -0.011 & 0.024 & $\nu$ & 0.194 & -0.006 & 0.004 \\
\hline & $\tau$ & 2.553 & 0.553 & 1.590 & $\tau$ & 2.523 & 0.523 & 0.429 \\
\hline
\end{tabular}

The estimated survival functions are displayed in Figure 4 by considering the AEs given in Table 1 for $n=100$, and considering the maximum and minimum values of the generated $x_{i}$ variable.

(a)

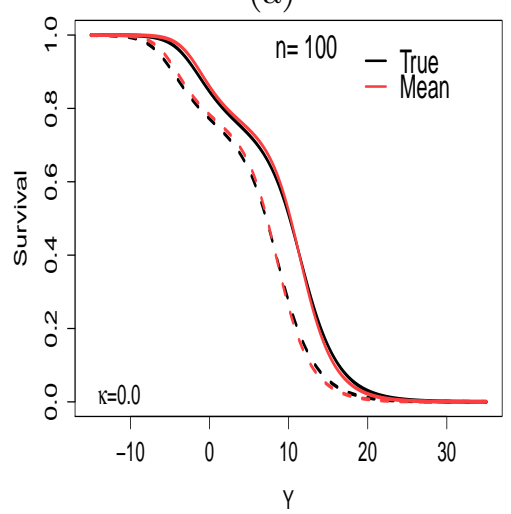

(b)

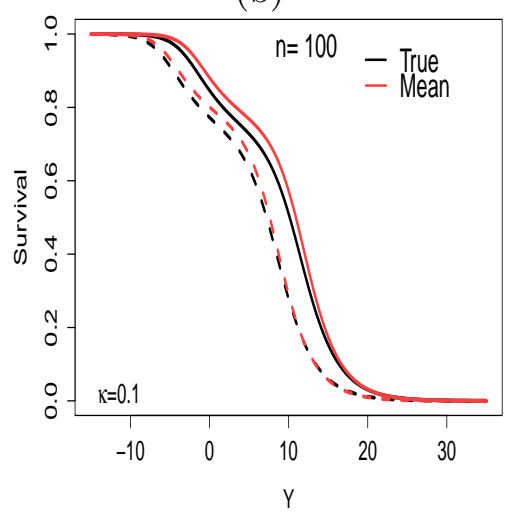

(c)

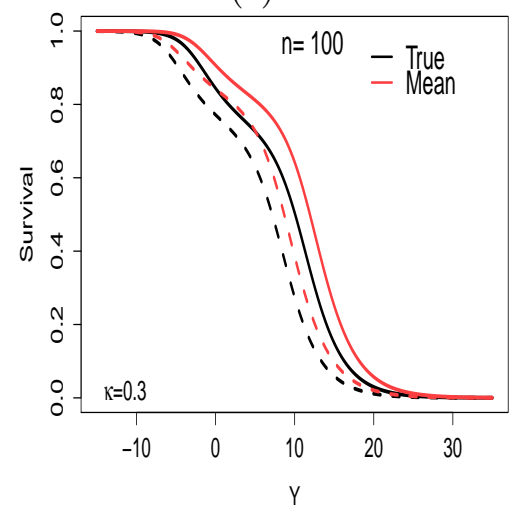

Figure 4: Some ESC survival functions at the true parameter values and at the AEs obtained in Table 1 , considering $n=100$ for the maximum and minimum of $x_{i}$ when: (a) $\kappa=0$; (b) $\kappa=0.1$; (c) $\kappa=0.3$. 


\section{ACCEPTED MANUSCRIPT}

\subsection{GAMLSS simulation}

For the GAMLSS, the true parameter values used in the data-generating process are $\mu_{i}=0.5+6 x_{i}$, $\sigma_{i}=\exp \left(1.5+0.6 x_{i}\right), \nu_{i}=\exp \left(-3.5+3 x_{i}\right)$ and $\tau_{i}=\exp \left(0.2+0.9 x_{i}\right)$. For each fit, the AEs, biases and MSEs are reported in Table 2.

Table 2: The AEs, biases and MSEs based on 2,000 simulations of the ESC regression model when $\beta_{01}=0.5, \beta_{11}=6, \beta_{02}=1.5, \beta_{12}=0.6, \beta_{03}=-3.5, \beta_{13}=3, \beta_{04}=0.2$ and $\beta_{14}=0.9$, for $n=50$ and 100 and under censoring percentages $\kappa=0.0,0.1$ and 0.3 .

\begin{tabular}{|c|c|c|c|c|c|c|c|c|}
\hline \multirow[b]{2}{*}{$\kappa$} & \multirow[b]{2}{*}{ Parameter } & \multicolumn{3}{|c|}{$n=50$} & \multirow[b]{2}{*}{ Parameter } & \multicolumn{3}{|c|}{$n=100$} \\
\hline & & $\mathrm{AE}$ & Bias & MSE & & $\mathrm{AE}$ & Bias & MSE \\
\hline \multirow[t]{8}{*}{0.0} & $\beta_{01}$ & 0.547 & 0.047 & 5.845 & $\beta_{01}$ & 0.471 & -0.029 & 2.647 \\
\hline & $\beta_{11}$ & 7.041 & 1.041 & 29.142 & $\beta_{11}$ & 6.756 & 0.756 & 13.629 \\
\hline & $\beta_{02}$ & 1.375 & -0.125 & 0.072 & $\beta_{02}$ & 1.414 & -0.086 & 0.030 \\
\hline & $\beta_{12}$ & 0.587 & -0.013 & 0.186 & $\beta_{12}$ & 0.571 & -0.029 & 0.089 \\
\hline & $\beta_{03}$ & -4.058 & -0.558 & 1.336 & $\beta_{03}$ & -3.861 & -0.361 & 0.536 \\
\hline & $\beta_{13}$ & 3.490 & 0.490 & 2.414 & $\beta_{13}$ & 3.273 & 0.273 & 1.061 \\
\hline & $\beta_{04}$ & 0.220 & 0.020 & 0.135 & $\beta_{04}$ & 0.228 & 0.028 & 0.061 \\
\hline & $\beta_{14}$ & 0.908 & 0.008 & 0.456 & $\beta_{14}$ & 0.895 & -0.005 & 0.211 \\
\hline \multirow[t]{8}{*}{0.1} & $\beta_{01}$ & 0.505 & 0.005 & 5.676 & $\beta_{01}$ & 0.546 & 0.046 & 2.632 \\
\hline & $\beta_{11}$ & 6.903 & 0.903 & 28.215 & $\beta_{11}$ & 6.664 & 0.664 & 16.902 \\
\hline & $\beta_{02}$ & 1.388 & -0.112 & 0.064 & $\beta_{02}$ & 1.446 & -0.054 & 0.025 \\
\hline & $\beta_{12}$ & 0.656 & 0.056 & 0.218 & $\beta_{12}$ & 0.597 & -0.003 & 0.098 \\
\hline & $\beta_{03}$ & -4.018 & -0.518 & 1.171 & $\beta_{03}$ & -3.797 & -0.297 & 0.457 \\
\hline & $\beta_{13}$ & 3.479 & 0.479 & 2.578 & $\beta_{13}$ & 3.248 & 0.248 & 0.969 \\
\hline & $\beta_{04}$ & 0.265 & 0.065 & 0.132 & $\beta_{04}$ & 0.309 & 0.109 & 0.063 \\
\hline & $\beta_{14}$ & 0.975 & 0.075 & 0.494 & $\beta_{14}$ & 0.865 & -0.035 & 0.211 \\
\hline \multirow[t]{8}{*}{0.3} & $\beta_{01}$ & 0.889 & 0.389 & 7.340 & $\beta_{01}$ & 0.636 & 0.136 & 3.020 \\
\hline & $\beta_{11}$ & 6.381 & 0.381 & 21.376 & $\beta_{11}$ & 6.319 & 0.319 & 9.264 \\
\hline & $\beta_{02}$ & 1.450 & -0.050 & 0.092 & $\beta_{02}$ & 1.482 & -0.018 & 0.040 \\
\hline & $\beta_{12}$ & 0.718 & 0.118 & 0.307 & $\beta_{12}$ & 0.753 & 0.153 & 0.183 \\
\hline & $\beta_{03}$ & -3.939 & -0.439 & 1.576 & $\beta_{03}$ & -3.807 & -0.307 & 0.640 \\
\hline & $\beta_{13}$ & 3.499 & 0.499 & 3.137 & $\beta_{13}$ & 3.478 & 0.478 & 1.580 \\
\hline & $\beta_{04}$ & 0.510 & 0.310 & 0.272 & $\beta_{04}$ & 0.508 & 0.308 & 0.155 \\
\hline & $\beta_{14}$ & 0.789 & -0.111 & 0.511 & $\beta_{14}$ & 0.790 & -0.110 & 0.206 \\
\hline
\end{tabular}

The estimated survival functions are displayed in Figure 5 and the AEs are listed in Table 2 for $n=100$, and considering the maximum and minimum values of the generated $x_{i}$ variable.

The results of the Monte Carlo study in Tables 1 and 2 indicate that the MSEs of the MLEs of the parameters decay toward zero when the sample size increases, as expected under first-order asymptotic theory. Note that the results of the GAMLSS simulation, presented in Table 2, should be interpreted by peers due to the fit of $\beta_{i k}$ influences the fit of $\beta_{j k}$. If $n$ increases, the AEs tend to be closer to the true parameter values. This fact supports that the asymptotic normal distribution provides an adequate approximation to the finite sample distribution of the MLEs. The normal approximation can oftentimes be improved by using bias adjustments to these estimators. In general, for the ESC 


\section{ACCEPTED MANUSCRIPT}

(a)

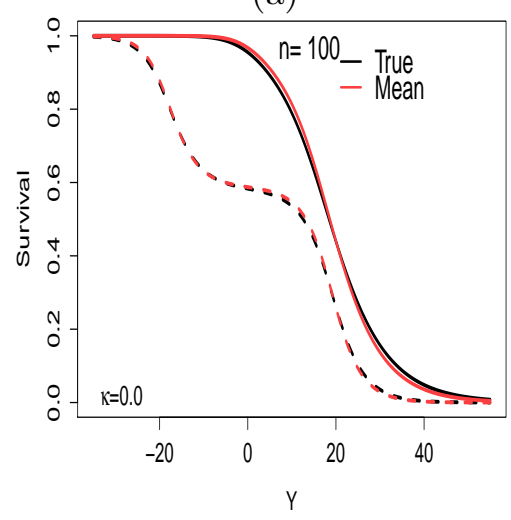

(b)

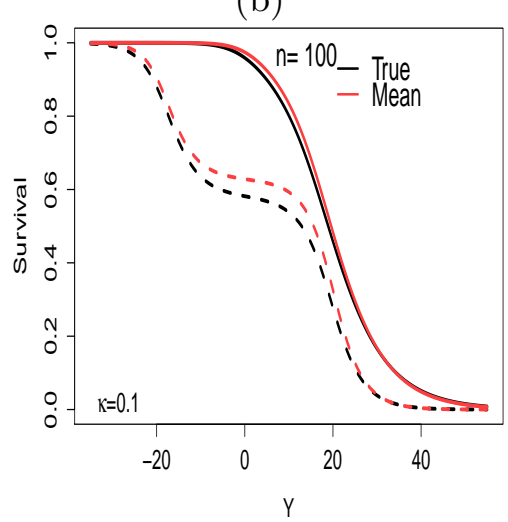

(c)

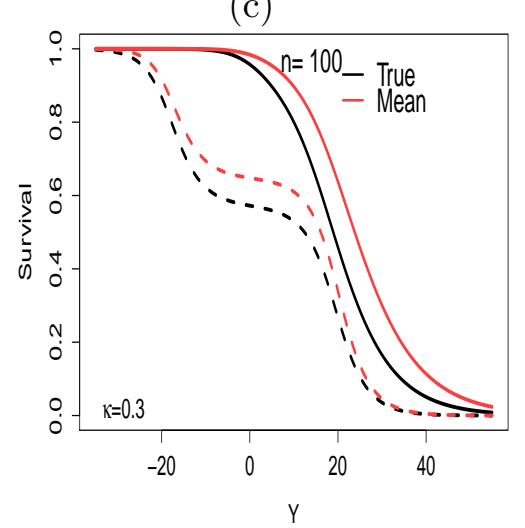

Figure 5: Some ESC survival functions at the true parameter values and at the AEs obtained in Table 2 , considering $n=100$ for the maximum and minimum of $x_{i}$ when: (a) $\kappa=0$; (b) $\kappa=0.1$; (c) $\kappa=0.3$.

regression models, the variances and MSEs increase when the censoring percentage increases. This fact can be noted in Figures 4 and 5 .

\section{Study of model misspecification}

To assess the behavior of the MLEs of the parameters in the ESC regression model when it is poorly specified, we carry out a Monte Carlo simulation study based on 1,000 replications using the GAMLSS. The logarithms of the lifetime data are generated from the $\log$-Weibull $(y, \mu, \sigma)$ and normal $(y, \mu, \sigma)$ heteroscedastic regression models (traditional models used in the survival analysis) for selected parameters $\mu=\beta_{01}+\beta_{11} x_{1}$ and $\sigma=\exp \left(\beta_{02}+\beta_{12} x_{1}\right)$, where the covariate $x_{i}$ is generated from a binomial $(n, 0.5)$ distribution. The censored indicators are generated randomly by fixing the censoring percentage. We consider the configuration with sample size $n=100, \beta_{01}=4.5, \beta_{11}=1.5, \beta_{02}=-1.5$, $\beta_{12}=1.5$ and censoring percentages of $\rho=0 \%, 10 \%$ and $30 \%$ to generate the samples. We fit the ESC regression model to each generated data set. The results of this study are given in Table 3, where we can note that an increasing in censoring percentage in general implies an increasing in the MSEs. There is a small sample bias in the estimation of the parameters of this regression model. Hence, it can provide consistent MLEs even when the data are generated from a different model.

Table 3: Mean estimates and MSEs (in parentheses) of the MLEs of the parameters in the log-Weibull and normal heteroscedastic regression models.

\begin{tabular}{cccc|ccc}
\hline \hline & \multicolumn{3}{c|}{ log-Weibull } & \multicolumn{3}{c}{ normal } \\
Parameter & $\rho=0 \%$ & $\rho=10 \%$ & $\rho=30 \%$ & $\rho=0 \%$ & $\rho=10 \%$ & $\rho=30 \%$ \\
\hline \hline$\beta_{01}$ & $4.510(0.005)$ & $4.526(0.006)$ & $4.553(0.009)$ & $4.452(0.006)$ & $4.467(0.006)$ & $44.488(0.006)$ \\
$\beta_{11}$ & $1.569(0.087)$ & $1.611(0.101)$ & $1.701(0.123)$ & $1.385(0.076)$ & $1.427(0.079)$ & $1.482(0.080)$ \\
$\beta_{02}$ & $-1.905(0.224)$ & $-1.838(0.275)$ & $-1.734(0.209)$ & $-1.744(0.086)$ & $-1.687(0.072)$ & $-1.545(0.025)$ \\
$\beta_{12}$ & $1.498(0.041)$ & $1.494(0.043)$ & $1.514(0.047)$ & $1.496(0.029)$ & $1.505(0.029)$ & $1.503(0.033)$ \\
$\nu$ & $1.207(-)$ & $1.271(-)$ & $1.280(-)$ & $1.084(-)$ & $1.122(-)$ & $1.150(-)$ \\
$\tau$ & $0.608(-)$ & $0.637(-)$ & $0.733(-)$ & $1.309(-)$ & $1.339(-)$ & $1.490(-)$ \\
\hline \hline
\end{tabular}




\section{ACCEPTED MANUSCRIPT}

\section{$6 \quad$ Sensitivity and residual analysis}

Since regression models are sensitive to the underlying model assumptions, performing a sensitivity analysis is strongly advisable. Cook (1986) used this idea to motivate the assessment of influence analysis. He suggested that more confidence can be put in a model, which is relatively stable under small modifications. The best known perturbation schemes are based on case-deletion (Cook and Weisberg, 1982), in which the effects of completely removing cases from the analysis are studied.

\subsection{Global influence}

A first tool to perform sensitivity analyses, as stated before, is by means of global influence starting from case-deletion. Case-deletion is a common approach to study the effect of dropping the $i$ th case from the data set. The case-deletion model for model (15) is given by

$$
y_{l}=\mu_{l}+\sigma_{l} z_{l}, \quad l=1, \ldots, n, \quad l \neq i,
$$

where the random error $Z_{l}$ has a density function $f\left(z_{l} ; \nu_{l}, \tau_{l}\right)$ given in (7). Of course, not always the explanatory variables will be modeling all parameters. For example, if we consider the class of location in (18), the case-deletion model reduces to

$$
y_{l}=\mu_{l}+\sigma z_{l}, \quad l=1, \ldots, n, \quad l \neq i,
$$

where the random error $Z_{l}$ has the density function $f\left(z_{l} ; \nu, \tau\right)$.

In the following, a quantity with subscript " $(i)$ " means the original quantity with the $i$ th case deleted. For model (18), the log-likelihood function of $\boldsymbol{\theta}$ is denoted by $l_{(i)}(\boldsymbol{\theta})$. Let $\hat{\boldsymbol{\theta}}_{(i)}^{T}=\left(\hat{\boldsymbol{\mu}}_{(i)}^{T}, \hat{\boldsymbol{\sigma}}_{(i)}^{T}, \hat{\boldsymbol{\nu}}_{(i)}^{T}\right.$, $\left.\hat{\boldsymbol{\tau}}_{(i)}^{T}\right)$ be the MLE of $\boldsymbol{\mu}, \boldsymbol{\sigma}, \boldsymbol{\nu}$ and $\boldsymbol{\tau}$ from $l_{(i)}(\boldsymbol{\theta})$. To assess the influence of the $i$ th case on the MLE $\hat{\boldsymbol{\theta}}^{T}=\left(\hat{\boldsymbol{\mu}}^{T}, \hat{\boldsymbol{\sigma}}^{T}, \hat{\boldsymbol{\nu}}^{T}, \hat{\boldsymbol{\tau}}^{T}\right)$, the basic idea is to compare the difference between $\hat{\boldsymbol{\theta}}_{(i)}$ and $\hat{\boldsymbol{\theta}}$. If deletion of a case seriously influences the estimates, for example changing the inference, more attention should be given to that case. Hence, if $\hat{\boldsymbol{\theta}}_{(i)}$ is far from $\hat{\boldsymbol{\theta}}$, then the $i$ th case is regarded as an influential observation. A first measure of the global influence is defined as the standardized norm of $\left(\hat{\boldsymbol{\theta}}_{(i)}-\hat{\boldsymbol{\theta}}\right)$, known as the generalized Cook distance, defined by

$$
G D_{i}(\boldsymbol{\theta})=\left(\hat{\boldsymbol{\theta}}_{(i)}-\hat{\boldsymbol{\theta}}\right)^{T}[-\ddot{\mathbf{L}}(\hat{\boldsymbol{\theta}})]\left(\hat{\boldsymbol{\theta}}_{(i)}-\hat{\boldsymbol{\theta}}\right) .
$$

Another alternative is to assess values $G D_{i}(\boldsymbol{\mu}), G D_{i}(\boldsymbol{\sigma}), G D_{i}(\boldsymbol{\nu})$ and $G D_{i}(\boldsymbol{\tau})$, which reveal the impact of the $i$ th observation on the estimates of $\boldsymbol{\mu}, \boldsymbol{\sigma}, \boldsymbol{\nu}$ and $\boldsymbol{\tau}$, respectively. Another popular measure of the difference between $\hat{\boldsymbol{\theta}}_{(i)}$ and $\hat{\boldsymbol{\theta}}$ is the likelihood distance defined by

$$
L D_{i}(\boldsymbol{\theta})=2\left[l(\hat{\boldsymbol{\theta}})-l\left(\hat{\boldsymbol{\theta}}_{(i)}\right)\right] .
$$

\subsection{Local influence}

Cook (1986) suggested to give weights to the observations instead of removing them. Local influence calculation can be carried out for model (15). If likelihood displacement $L D(\boldsymbol{\omega})=2\{l(\hat{\boldsymbol{\theta}})-l(\hat{\boldsymbol{\theta}} \boldsymbol{\omega})\}$ is used, where $\hat{\boldsymbol{\theta}}_{\boldsymbol{\omega}}$ denotes the MLE under the perturbed model, the normal curvature for $\boldsymbol{\theta}$ in the 


\section{ACCEPTED MANUSCRIPT}

direction $\mathbf{d},\|\mathbf{d}\|=1$, is given by $C_{\mathbf{d}}(\boldsymbol{\theta})=2\left|\mathbf{d}^{T} \boldsymbol{\Delta}^{T} \ddot{\mathbf{L}}_{\theta \theta}^{-1} \boldsymbol{\Delta} \mathbf{d}\right|$, where $\boldsymbol{\Delta}$ is a $p \times n$ matrix that depends on the perturbation scheme, whose elements are given by $\Delta_{v i}=\partial^{2} l(\boldsymbol{\theta} \mid \boldsymbol{\omega}) / \partial \theta_{v} \partial \omega_{i}, i=1, \ldots, n$ and $v=1, \ldots, p$, evaluated at $\hat{\boldsymbol{\theta}}$ and $\boldsymbol{\omega}_{0}$, and $\boldsymbol{\omega}_{0}$ is the no perturbation vector. We can also calculate normal curvatures $C_{\mathbf{d}}(\boldsymbol{\mu}), C_{\mathbf{d}}(\boldsymbol{\sigma}), C_{\mathbf{d}}(\boldsymbol{\nu})$ and $C_{\mathbf{d}}(\boldsymbol{\tau})$ to perform various index plots, for instance, the index plot of $\mathbf{d}_{\text {max }}$, the eigenvector corresponding to $C_{\mathbf{d}_{\text {max }}}$, the largest eigenvalue of the matrix $\mathbf{B}=-\boldsymbol{\Delta}^{T} \ddot{\mathbf{L}}_{\theta \theta}^{-1} \boldsymbol{\Delta}$ and the index plots of $C_{\mathbf{d}_{i}}(\boldsymbol{\mu}), C_{\mathbf{d}_{i}}(\boldsymbol{\sigma}), C_{\mathbf{d}_{i}}(\boldsymbol{\nu})$ and $C_{\mathbf{d}_{i}}(\boldsymbol{\tau})$, named the total local influence (Lesaffre and Verbeke, 1998), where $\mathbf{d}_{i}$ denotes an $n \times 1$ vector of zeros with one at the $i$ th position. Thus, the curvature in the direction $\mathbf{d}_{i}$ takes the form $C_{i}=2\left|\boldsymbol{\Delta}_{i}^{T} \ddot{\mathbf{L}}_{\theta \theta}^{-1} \boldsymbol{\Delta}_{i}\right|$, where $\boldsymbol{\Delta}_{i}^{T}$ denotes the $i$ th row of $\boldsymbol{\Delta}$. It is usual to point out those cases such that $C_{i} \geq 2 \bar{C}$, where $\bar{C}=\frac{1}{n} \sum_{i=1}^{n} C_{i}$. In some situations, the information of the matrix $\mathbf{B}$ may be contained not only in the first eigenvalue, then an alternative influence measure for the $i$ th observation is $U_{i}=\sum_{k=1}^{n_{1}} \lambda_{k} e_{k i}^{2}$, where $\left\{\left(\lambda_{k}, \mathbf{e}_{k}\right) \mid k=\right.$ $1, \ldots, n\}$ are the eigenvalue-eigenvector pairs of $\mathbf{B}$ with $\lambda_{1} \geq \cdots \geq \lambda_{n_{1}} \geq \lambda_{n_{1}+1}=\cdots=\lambda_{n}=0$ and $\left\{\mathbf{e}_{k}=\left(e_{k 1}, \ldots, e_{k n}\right)^{T}\right\}$ is the associated orthonormal basis. Zhu et al. (2007) studied the influence measure $u_{i}$ systematically under a case-weight perturbation. Thus, this influence measure expresses local sensitivity to the log-likelihood of the perturbations.

Next, we obtain under model (15) and log-likelihood function (17), for three perturbation schemes, the matrix

$$
\boldsymbol{\Delta}=\left(\boldsymbol{\Delta}_{v i}\right)_{p \times n}=\left(\frac{\partial^{2} l(\boldsymbol{\theta} \mid \boldsymbol{\omega})}{\partial \theta_{v} \partial \boldsymbol{\omega}_{i}}\right)_{p \times n}, \quad v=1, \ldots, p \quad \text { and } \quad i=1, \ldots, n \text {. }
$$

\subsubsection{Case-weight perturbation}

Consider the vector of weights $\boldsymbol{\omega}=\left(\omega_{1}, \ldots, \omega_{n}\right)^{T}$, where $0 \leq \omega_{i} \leq 1$. A perturbed log-likelihood function, allowing different weights for different observations, can be defined in the form $l(\boldsymbol{\theta} \mid \boldsymbol{\omega})=$ $\sum_{i \in F} w_{i} \log f\left(y_{i}\right)+\sum_{i \in C} w_{i} \log S\left(y_{i}\right)$. Also, let $w_{0}=(1, \ldots, 1)^{T}$ be the vector of no perturbation such that $l\left(\boldsymbol{\theta} \mid w_{0}\right)=l(\boldsymbol{\theta})$. In this case, the log-likelihood function takes the form

$l(\boldsymbol{\theta} \mid \boldsymbol{\omega})=\sum_{i \in F} \omega_{i}\left[-\log d_{i}+\left(\tau_{i}-1\right) \log \left(h_{i}\right)+\log \cosh \left(z_{i}\right)+\log \left(\tau_{i} \nu_{i}\right)-\log \left(\sigma_{i} \pi\right)\right]+\sum_{i \in C} \omega_{i} \log \left[1-h_{i}^{\tau_{i}}\right]$,

where $h_{i}=0.5+\pi^{-1} \arctan \left[\nu_{i} \sinh \left(z_{i}\right)\right]$ and $d_{i}=\left[1+\nu_{i}^{2} \sinh ^{2}\left(z_{i}\right)\right]$. The matrix $\boldsymbol{\Delta}=\left(\boldsymbol{\Delta}_{\boldsymbol{\mu}}^{T}, \boldsymbol{\Delta}_{\boldsymbol{\sigma}}^{T}, \boldsymbol{\Delta}_{\boldsymbol{\nu}}^{T}\right.$, $\left.\boldsymbol{\Delta}_{\boldsymbol{\tau}}^{T}\right)^{T}$ can be calculated numerically.

\subsubsection{Response perturbation}

Since the values of $y_{i}$ have different variances, they require a scaling of the perturbation vector $\boldsymbol{\omega}$ by an estimator of the standard deviation of $y_{i}$. We shall consider that each $y_{i}$ is perturbed as $y_{i w}=y_{i}+\omega_{i} S_{y}$, where $S_{y}$ is a scale factor that may be estimated by the standard deviation of $y$ and $\omega_{i} \in \mathbf{R}$. Then, the perturbed log-likelihood function becomes

$$
l(\boldsymbol{\theta})=\sum_{i \in F}\left[-\log d_{i}^{*}+\log \cosh \left(z_{i}^{*}\right)+\left(\tau_{i}-1\right) \log \left(h_{i}^{*}\right)+\log \left(\tau_{i} \nu_{i}\right)-\log \left(\sigma_{i} \pi\right)\right]+\sum_{i \in C} \log \left(1-h_{i}^{* \tau_{i}}\right),
$$

where $h_{i}^{*}=0.5+\pi^{-1} \arctan \left[\nu_{i} \sinh \left(z_{i}^{*}\right)\right], d_{i}^{*}=\left[1+\nu_{i}^{2} \sinh ^{2}\left(z_{i}^{*}\right)\right]$ and $z_{i}^{*}=\left(y_{i}+\omega_{i} S_{y}-\mu_{i}\right) / \sigma_{i}$. The matrix $\boldsymbol{\Delta}=\left(\boldsymbol{\Delta}_{\boldsymbol{\mu}}^{T}, \boldsymbol{\Delta}_{\boldsymbol{\sigma}}^{T}, \boldsymbol{\Delta}_{\boldsymbol{\nu}}^{T}, \boldsymbol{\Delta}_{\boldsymbol{\tau}}^{T}\right)^{T}$ can be calculated numerically. 


\section{ACCEPTED MANUSCRIPT}

\subsubsection{Explanatory variable perturbation}

We consider an additive perturbation on a particular continuous explanatory variable, namely $x_{1}[i, t]$, by setting $x_{1}[i, t \omega]=x_{1}[i, t]+\omega_{i} S_{x}$, where $S_{x}$ is a scaled factor, $\omega_{i} \in \mathbf{R}$. Note that the explanatory variable $x_{1}[i, t]$ is related only to the location parameter $\mu$. However, this perturbation scheme can be extended by considering different numbers of explanatory variables for different parameters.

This perturbation scheme leads to the perturbed log-likelihood function

$l(\boldsymbol{\theta})=\sum_{i \in F}\left[-\log d_{i}^{\star}+\log \cosh \left(z_{i}^{\star}\right)+\left(\tau_{i}-1\right) \log \left(h_{i}^{\star}\right)+\log \left(\tau_{i} \nu_{i}\right)-\log \left(\sigma_{i} \pi\right)\right]+\sum_{i \in C} \log \left(1-h_{i}^{\star \tau_{i}}\right)$,

where $h_{i}^{\star}=0.5+\pi^{-1} \arctan \left[\nu_{i} \sinh \left(z_{i}^{\star}\right)\right], d_{i}^{\star}=\left[1+\nu_{i}^{2} \sinh ^{2}\left(z_{i}^{\star}\right)\right], z_{i}^{\star}=\left(y_{i}-\mu_{i}^{\star}\right) / \sigma_{i}$ and $\mu_{i}^{\star}=$ $\beta_{01}+\beta_{11} x_{1}[i, 2], \ldots, \beta_{t 1}\left(x_{1}[i, t]+\omega_{i} S_{x} \ldots, \beta_{p_{1} 1} x_{1}\left[p_{1}, 1\right]\right)$. The matrix $\boldsymbol{\Delta}=\left(\boldsymbol{\Delta}_{\boldsymbol{\mu}}^{T}, \boldsymbol{\Delta}_{\boldsymbol{\sigma}}^{T}, \boldsymbol{\Delta}_{\boldsymbol{\nu}}^{T}, \boldsymbol{\Delta}_{\boldsymbol{\tau}}^{T}\right)^{T}$ can be calculated numerically.

\subsection{Residual Analysis}

In order to study departures from the error assumption and the presence of outliers, we consider the martingale residual proposed by Barlow and Prentice (1998) and the transformation of this residual. More details may be found in Ortega et al. (2003).

The martingale residuals, recommended in counting processes, are defined by $r_{M_{i}}=\delta_{i}+\log \left[S\left(y_{i} ; \hat{\boldsymbol{\beta}}\right)\right]$, where $\delta_{i}=0,1$ denotes a censored and uncensored observation, respectively, and $S\left(y_{i} ; \hat{\boldsymbol{\beta}}\right)$ denotes the survival function of $Y$ discussed in Section 1. Recently, several authors have studied the martingale residual for some regression models. Silva et al. (2008) proposed using the martingale residual for the log-Burr XII regression model considering censored data, Cancho et al. (2009) studied the residuals for the log-exponentiated-Weibull regression model with cure rate, Ortega et al. (2014) derived the martingale residual for the odd Weibull regression models for censored data, among others.

This residual was introduced in the counting process (Fleming and Harrington, 1991) and can be expressed in the ESC regression models as

$$
r_{M_{i}}= \begin{cases}1-\hat{\tau}_{i} \log (2 \pi)+\log \left[(2 \pi)^{\hat{\tau}_{i}}-\left\{\pi+2 \arctan \left[\hat{\nu}_{i} \sinh \left(\hat{z}_{i}\right)\right]\right\}^{\hat{\tau}_{i}}\right] & \text { if } i \in F \\ -\hat{\tau}_{i} \log (2 \pi)+\log \left[(2 \pi)^{\hat{\tau}_{i}}-\left\{\pi+2 \arctan \left[\hat{\nu}_{i} \sinh \left(\hat{z}_{i}\right)\right]\right\}^{\hat{\tau}_{i}}\right] & \text { if } i \in C,\end{cases}
$$

where $\hat{z}_{i}=\left(y_{i}-\hat{\mu}_{i}\right) / \hat{\sigma}_{i}, \mu_{i}=\hat{\beta}_{01}+\ldots+x_{1}\left[i, p_{1}+1\right] \hat{\beta}_{p_{1} 1}, \sigma_{i}=\exp \left(\hat{\beta}_{02}+\ldots+x_{2}\left[i, p_{2}+1\right] \hat{\beta}_{p_{2} 2}\right)$, $\nu_{i}=\exp \left(\hat{\beta}_{03}+\ldots+x_{3}\left[i, p_{3}+1\right] \hat{\beta}_{p_{3} 3}\right)$ and $\tau_{i}=\exp \left(\hat{\beta}_{04}+\ldots+x_{4}\left[i, p_{4}+1\right] \hat{\beta}_{p_{4} 4}\right)$. In fact, $r_{M_{i}}$ ranges from a maximum value +1 and minimum value $-\infty$. A disadvantage of the martingale residual is that the distribution of $r_{M_{i}}$ is markedly skewed, and so it fails to have similar properties to those of the normal distribution. Suitable transformations to achieve a more normal shaped form would be more appropriate for residual analysis.

Another possibility is to use a transformation of the martingale residual based on the deviance residuals for the Cox model in the case of no time-dependent covariates (Therneau et al., 1990). We shall use this transformation of the martingale residual in order to have a new residual symmetrically distributed around zero. A more extensive examination of this residual is given by Leiva et al. (2007) 


\section{ACCEPTED MANUSCRIPT}

and Ortega et al. (2008). Thus, a martingale-type residual for the ESC regression model can be expressed as

$$
r_{D_{i}}=\operatorname{sign}\left(r_{M_{i}}\right)\left\{-2\left[r_{M_{i}}+\delta_{i} \log \left(\delta_{i}-r_{M_{i}}\right)\right]\right\}^{1 / 2}
$$

where $r_{M_{i}}$ is defined in equation (19) for $i \in F\left(\delta_{i}=1\right)$ or $i \in C\left(\delta_{i}=0\right)$.

For uncensored data, we can use the diagnostic tools in the gamlss package. The first technique consists in the normalized randomized quantile residuals (Dunn and Smyth, 1996) given by $\hat{r}_{i}=$ $\Phi^{-1}\left(u_{i}\right)$, where $\Phi^{-1}(\cdot)$ is the inverse cdf of a standard normal variate and $u_{i}=F\left(y_{i} \mid \hat{\boldsymbol{\theta}}_{i}\right)$.

The second technique already known in the literature is the normal probability plot with envelope. Atkinson (1985) suggested the construction of envelopes to enable better interpretation of the normal probability plots of the residuals. Such envelopes are simulated confidence bands that contain the residuals, such that if the model is well fitted, the majority of points will be within these bands and randomly distributed. The construction of the confidence bands follows the steps:

- Fit the proposed model, we evaluate the normalized randomized quantile residuals $\hat{r}_{i}$;

- Simulate $k$ samples of size $n$ of the response variable using the fitted model;

- For each sample, we compute the residuals $\hat{r}_{i j}, j=1,2, \ldots, k$ and $i=1,2, \ldots, n$;

- Arrange each group of $n$ residuals in rising order to obtain $\hat{r}_{(i) j}$;

- For each $i$, obtain the minimum and maximum $\hat{r}_{(i) j}$, namely:

$$
r_{(i) I}=\min \left\{r_{(i) j}: 1 \leq j \leq k\right\} \text { and } r_{(i) S}=\max \left\{r_{(i) j}: 1 \leq j \leq k\right\}
$$

- Include the minimum and maximum together with the values of $\hat{r}_{i}$ against the expected percentiles of the standard normal distribution.

The minimum and maximum values of $\hat{r}_{(i) j}$ define the envelope. If the model under study is correct, the observed values of $\hat{r}_{i}$ should be inside the bands and distributed randomly.

\section{Applications}

In this section, we provide two applications to real data to illustrate the flexibility of the ESC regression model. The computations are performed using the gamlss subroutine in the $\mathrm{R}$ software and the script is described in the Appendix. For the first data set, we prove empirically the flexibility of the new regression model when all parameters are modeled by explanatory variables (complete model). For the second data set, we present an application, where the scale and skewness parameters are modeled by explanatory variables. For both applications we provide the goodness-of-fit statistics Akaike information criterion (AIC) and Bayesian information criterion (BIC). The computational codes for the applications in subsections 7.1 and 7.2 are available available on the Web at http://goo.gl/zANZuz and http://goo.gl/ZBf8R8, respectively. 


\section{ACCEPTED MANUSCRIPT}

\subsection{Shrimp data}

Consider the data on biometric measurements in shrimps of farfantepenaeus brasiliensis species. These data were obtained from three regions of the Rio Grande do Norte state in Brazil, for which the objective was to relate the weights of the shrimps in each region. The importance of characterizing the weights of shrimps per region is discussed by Pinheiro (2008).

To exemplify the new propose, we consider the full sample $(n=120)$, where the response variable $t_{i}$ represents the $i$ th shrimp weight in grams and the three groups of region are defined by dummy variables: Baia formosa $\left(x_{i 1}=0\right.$ and $\left.x_{i 2}=0\right)$, Diogo Lopes $\left(x_{i 1}=1\right.$ and $\left.x_{i 2}=0\right)$ and Touros $\left(x_{i 1}=0\right.$ and $\left.x_{i 2}=1\right)$. Let the random variable $y_{i}=\log \left(t_{i}\right)$ have the ESC distribution (5). As a preliminary analysis, we note that the explanatory variable region affects the location, scale, bimodality and asymmetry parameters. This fact can easily be observed in Figure 6.

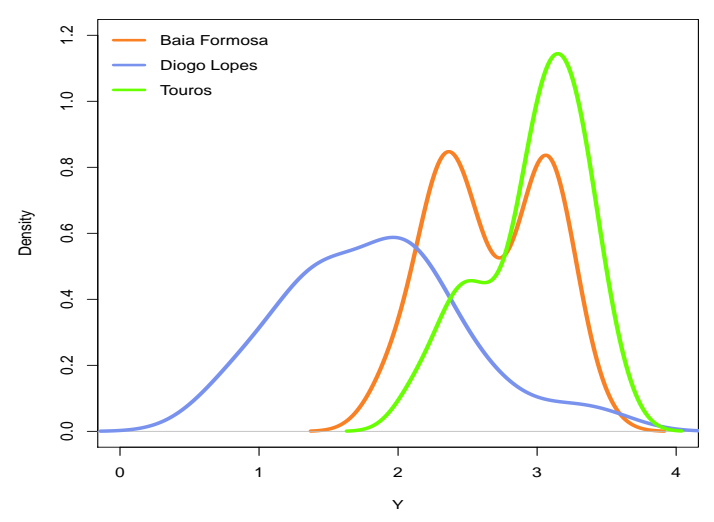

Figure 6: The empirical density of $Y$ in the different regions.

Next, we present results by fitting the model

$$
y_{i}=\mu_{i}+\sigma_{i} z_{i}
$$

where $z_{i}$ has density function (7) and the model parameters are defined by

$$
\begin{aligned}
& \mu_{i}=\beta_{01}+\beta_{11} x_{i 1}+\beta_{21} x_{i 2}, \quad \sigma_{i}=\exp \left(\beta_{02}+\beta_{12} x_{i 1}+\beta_{22} x_{i 2}\right), \\
& \nu_{i}=\exp \left(\beta_{03}+\beta_{13} x_{i 1}+\beta_{23} x_{i 2}\right) \quad \text { and } \quad \tau_{i}=\exp \left(\beta_{04}+\beta_{14} x_{i 1}+\beta_{24} x_{i 2}\right) \text {. }
\end{aligned}
$$

Table 4 provides the MLEs, their approximate standard errors and $p$-values, all quantities obtained from the fitted ESC regression model. The values of the goodness-of-fit statistics are $A I C=142.9$ and $B I C=176.3$. The results in Table 4 reveal that the explanatory variable region should be used to model the location, scale, bimodality and skewness parameters at the $5 \%$ level. Therefore, we can conclude that for each region, the weights of shrimps have different forms (bimodal and unimodal), different location scales and asymmetry, and then they can not be fitted only with a location model.

\subsubsection{Global influence analysis}

Here, we compute the case deletion measures $G D_{i}(\boldsymbol{\theta})$ and $L D_{i}(\boldsymbol{\theta})$ for the shrimp data. The results of such influence measure index plots are displayed in Figure 7 . We may note that the 62 th observation is a possible influential observation. 


\section{ACCEPTED MANUSCRIPT}

Table 4: MLEs of the parameters and their approximate standard errors from the fitted ESC regression model to the shrimp data.

\begin{tabular}{cccccccc}
\hline Parameter & Estimate & SE & $p$-value & Parameter & Estimate & SE & $p$-value \\
\hline$\beta_{01}$ & 2.721 & 0.034 & $<0.001$ & $\beta_{03}$ & -2.616 & 0.613 & $<0.001$ \\
$\beta_{11}$ & -1.163 & 0.398 & 0.004 & $\beta_{13}$ & 2.059 & 0.777 & 0.009 \\
$\beta_{21}$ & 0.594 & 0.091 & $<0.001$ & $\beta_{23}$ & 2.425 & 0.754 & 0.001 \\
$\beta_{02}$ & -2.235 & 0.175 & $<0.001$ & $\beta_{04}$ & -0.189 & 0.232 & 0.416 \\
$\beta_{12}$ & 1.223 & 0.387 & 0.002 & $\beta_{14}$ & 0.655 & 0.713 & 0.360 \\
$\beta_{22}$ & -0.057 & 0.495 & 0.908 & $\beta_{24}$ & -1.165 & 0.595 & 0.052 \\
\hline
\end{tabular}

(a)

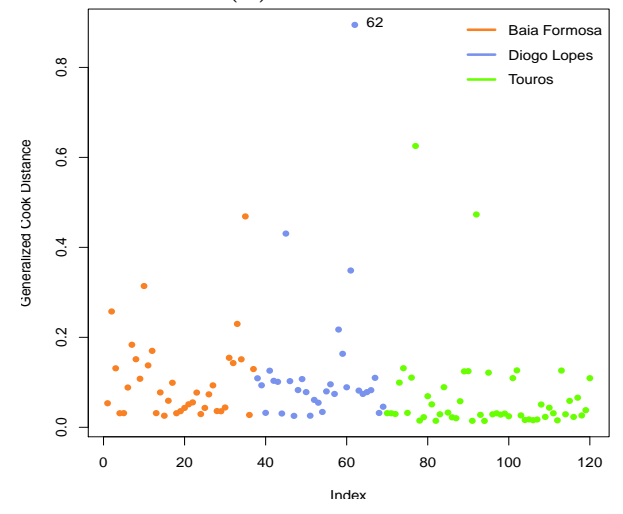

(b)

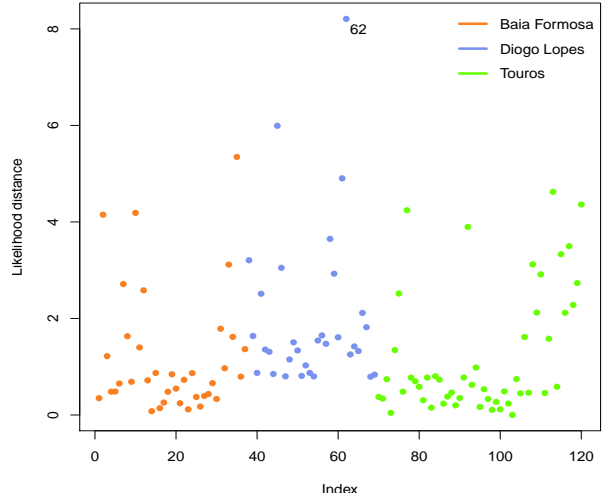

Figure 7: Index plots for $\boldsymbol{\theta}$ : (a) $G D_{i}(\boldsymbol{\theta})$ (Generalized Cook's Distance) and (b) $L D_{i}(\boldsymbol{\theta})$ (Likelihood Distance).

\subsubsection{Local influence analysis}

In this section, we perform the local influence analysis for the shrimp data using the ESC regression model.

\section{Case-weight perturbation}

By applying the local influence methodology, where the case-weight perturbation is used, the four largest eigenvalues of the matrix $\mathbf{B}$ are 1.65, 1.64, 1.26 and 1.12. Figure 8 displays the index plots of the $U_{i}$ measure and the total influence $C_{i}$. These plots reveal that the 62 th observation also appears as possible influential observation.

\section{Response perturbation}

Next, the influence of perturbations in the observed times is analyzed. Here, we adopt the $U_{i}$ measure instead of $\mathbf{d}_{\max }$ because the first eight eigenvalues are large. Figure 9 displays the index plot of the $U_{i}$ measure and the total local influence $C_{i}$.

Under the sensitivity analysis, we note that the 62 th observation once more appears as a possible influential point. In fact, this shrimp has the largest weight for Diogo Lopes region, being very different from the other measurements. The shrimps detected as possible influential observations in Figure 9 represent the measurements $y_{105}=2.89$ and $y_{107}=2.88$ of the Touro region. Combining with the plots of Figure 6, we can note that these two shrimps stabilize the growth of the density. 


\section{ACCEPTED MANUSCRIPT}
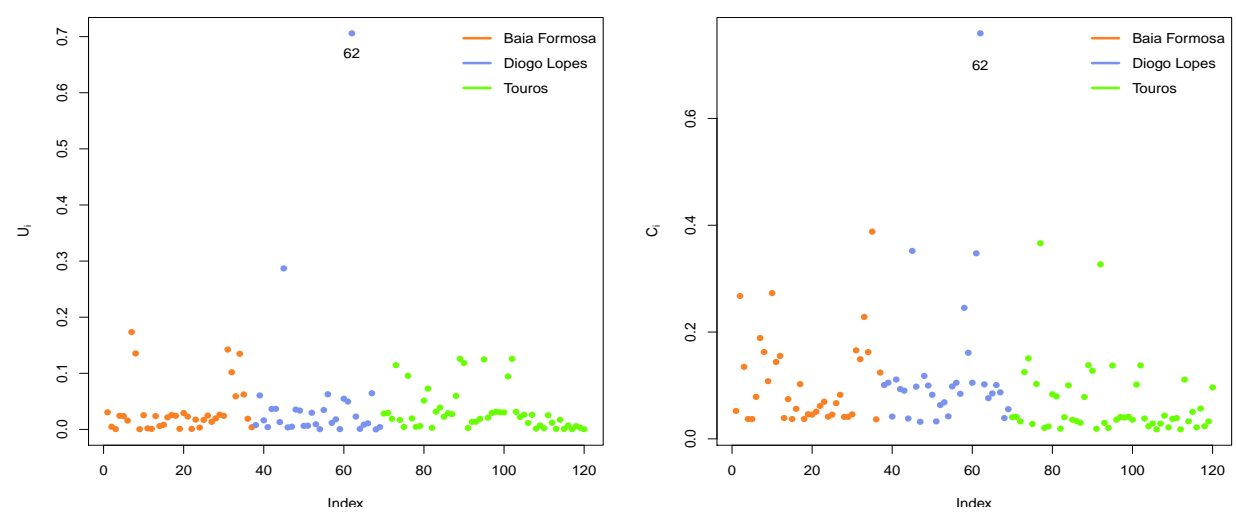

Figure 8: Index plots for $\boldsymbol{\theta}$ (case-weight perturbation): (a) $\mathbf{d}_{\max }$ and (b) total local influence.
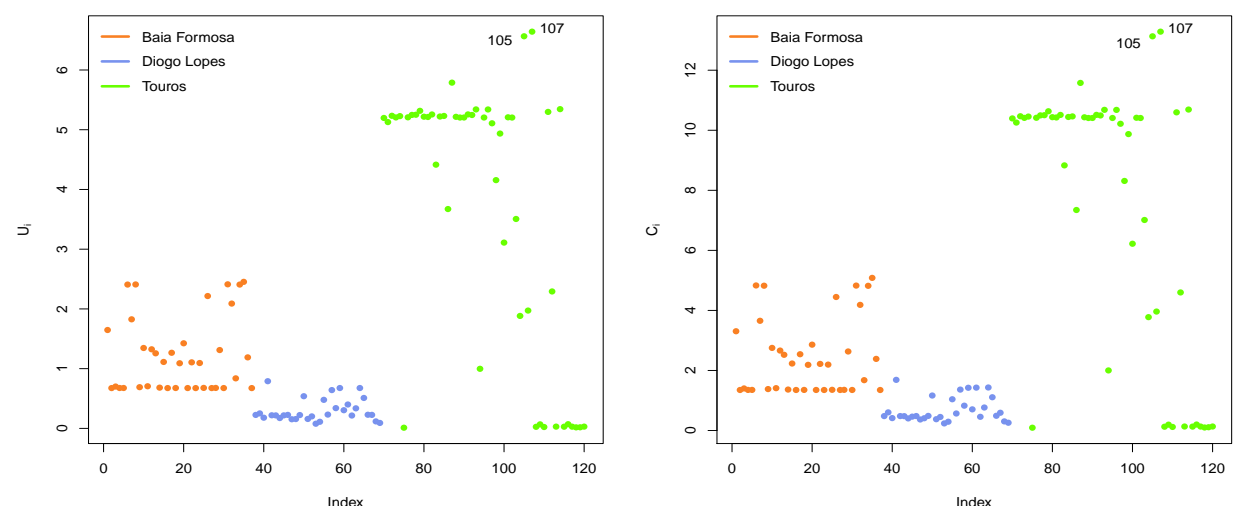

Figure 9: Index plots for $\boldsymbol{\theta}$ (response perturbation): (a) $\mathbf{d}_{\max }$ and (b) total local influence.

\subsubsection{Residual analysis}

In order to detect possible outlying observations as well as departures from the assumptions made for the ESC regression model, we present in Figure 10 the index plot as well as the normal probability plot with generated confidence band for the quantile residual. Note that the quantile residual seems to follow approximately a normal distribution, thus indicating a suitable fitted model. Note that the observations detected in the influence analysis are not detected in the residual analysis.

In order to assess whether the model fits the data appropriately, the empirical cdf and estimated cdf of the ESC regression model are plotted in Figure 11 for different regions. We conclude that the Exp-ESC regression model provides a very good fit to the shrimp data.

\subsection{Entomology data}

In the second application, we take a data set from a study carried out at the Department of Entomology of the Luiz de Queiroz School of Agriculture, University of São Paulo. Such study aims to assess the longevity of the mediterranean fruit fly (ceratitis capitata), which is considered a pest in agriculture. Instead of using an insecticide, Silva et al. (2013) conducted a study using small portions of food containing substances extracted from a tree called "neem". The experiment was completely randomized with eleven treatments, consisting of different extracts of the neem tree at concentrations 


\section{ACCEPTED MANUSCRIPT}

(a)

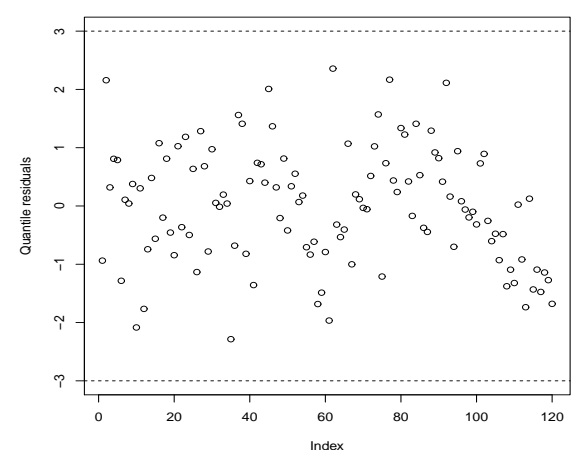

(b)

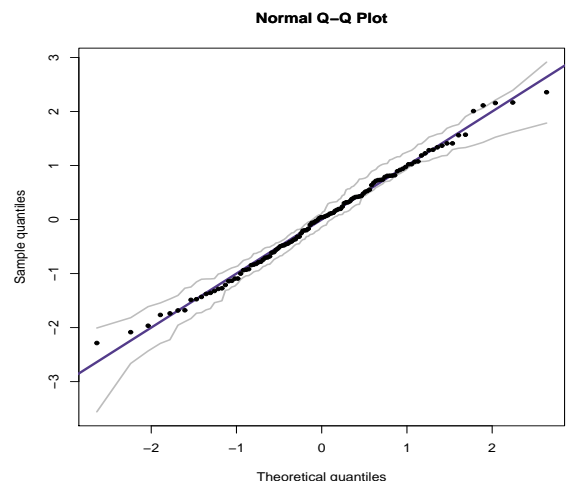

Figure 10: (a) Index plot of the quantile residuals for the shrimp data. (b) Normal probability plot with envelope for the quantile residuals from the fitted ESC regression model to the shrimp data.

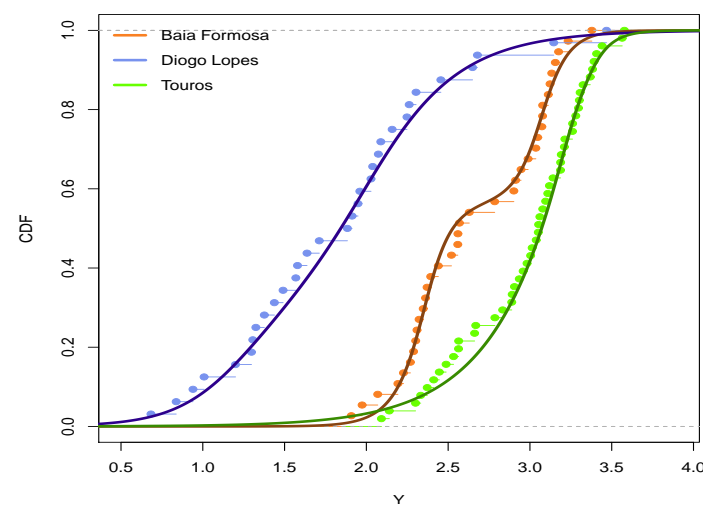

Figure 11: Estimated cumulative fitted values from the ESC fitted model to the shrimp data.

of 39,225 and $888 \mathrm{ppm}$, where the response variable is the lifetime of the adult flies in days after exposure to the treatments. The experimental period was set at 51 days, so that the numbers of larvae that survived beyond this period are considered as censored observations. From the results of the experiment, these eleven treatments are allocated into two groups, namely:

- Group 1: Control 1 (deionized water); Control 2 (acetone - 5\%); aqueous extract of seeds (AES) (39 ppm); AES (225 ppm); AES (888 ppm); methanol extract of leaves (MEL) (225 ppm); MEL (888 ppm); and dichloromethane extract of branches (DMB) (39 ppm).

- Group 2: MEL (39 ppm); DMB (225ppm) and DMB (888 ppm).

Let $t_{i}$ be the lifetime of ceratitis capitata adults in days, $\delta_{i}$ the censoring indicator and $x_{i 1}$ the dummy variable indicating the groups ( $0=$ group 1 and $1=$ group 2$)$. In a preliminary analysis, we note that only the scale and skewness parameters require explanatory variables. Next, we present results by fitting the model

$$
y_{i}=\beta_{01}+\sigma_{i} z_{i}
$$




\section{ACCEPTED MANUSCRIPT}

where $z_{i}$, for $i=1, \ldots, 172$, has density function $f\left(z_{i} ; \nu, \tau_{i}\right)$ given by $(7)$ and the model parameters are given by

$$
\mu_{i}=\beta_{01}, \quad \sigma_{i}=\exp \left(\beta_{02}+\beta_{12} x_{i 1}\right), \quad \nu_{i}=\exp \left(\beta_{03}\right) \quad \text { and } \quad \tau_{i}=\exp \left(\beta_{04}+\beta_{14} x_{i 1}\right) .
$$

Table 5 provides the MLEs, their approximate standard errors and $p$-values obtained from the fitted ESC regression model. We can conclude that the explanatory variable group should be used to model the scale and skewness parameters at the $1 \%$ level. The goodness-of-fit statistics obtained are $A I C=309.3$ and $B I C=328.2$. Recently, Cordeiro et al. (2015) fitted the log-generalized Weibulllog-logistic (LGW-LL) to these data and obtained the statistics $A I C=341$ and $B I C=357$. We conclude that the ESC regression model provides a good fit to these data.

Table 5: MLEs of the parameters and their approximate standard errors from the fitted ESC regression model to the entomology data.

\begin{tabular}{cccccccc}
\hline Parameter & Estimate & $\mathrm{SE}$ & $p$-value & Parameter & Estimate & $\mathrm{SE}$ & $p$-value \\
\hline$\beta_{01}$ & 3.013 & 0.024 & $<0.001$ & $\beta_{03}$ & 1.218 & 0.112 & $<0.001$ \\
$\beta_{02}$ & -0.012 & 0.119 & 0.913 & $\beta_{04}$ & 0.100 & 0.085 & 0.242 \\
$\beta_{12}$ & -0.895 & 0.234 & $<0.001$ & $\beta_{14}$ & -0.893 & 0.175 & $<0.001$ \\
\hline
\end{tabular}

\subsubsection{Global influence analysis}

Here, we compute the case deletion measures $G D_{i}(\boldsymbol{\theta})$ and $L D_{i}(\boldsymbol{\theta})$ for the entomology data. The results of such influence measure index plots are displayed in Figure 12. Based on these plots, we note that the cases 92 and 133 are possibly influential observations.

(a)

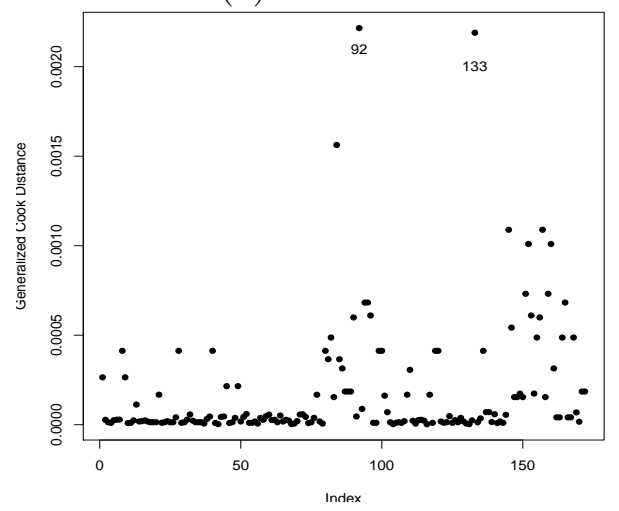

(b)

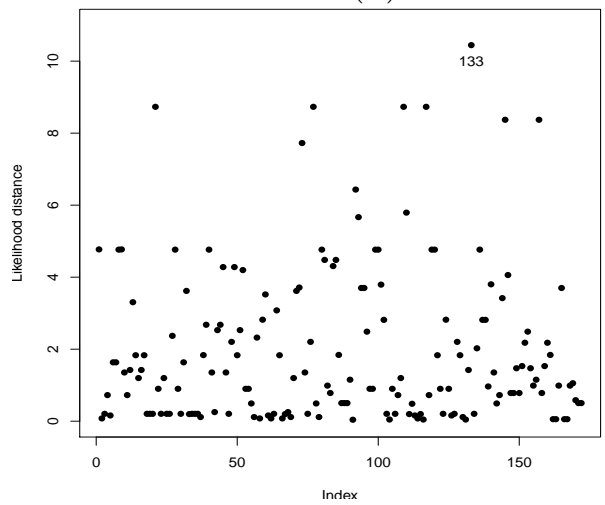

Figure 12: Index plots for $\boldsymbol{\theta}$ : (a) $G D_{i}(\boldsymbol{\theta})$ (Generalized Cook's Distance) and (b) $L D_{i}(\boldsymbol{\theta})$ (Likelihood Distance).

\subsubsection{Local influence analysis}

\section{Case-weight perturbation}




\section{ACCEPTED MANUSCRIPT}

By applying the local influence methodology, where case-weight perturbation is applied, we obtain $C_{\mathbf{d}_{\text {max }}}=1.15$ as the maximum curvature. Figure 13 display the index plots of the eigenvector corresponding to $\mathbf{d}_{\max }$ and the total influence $C_{i}$. We may conclude that the observations 145 and 157 present larger influence.

(a)

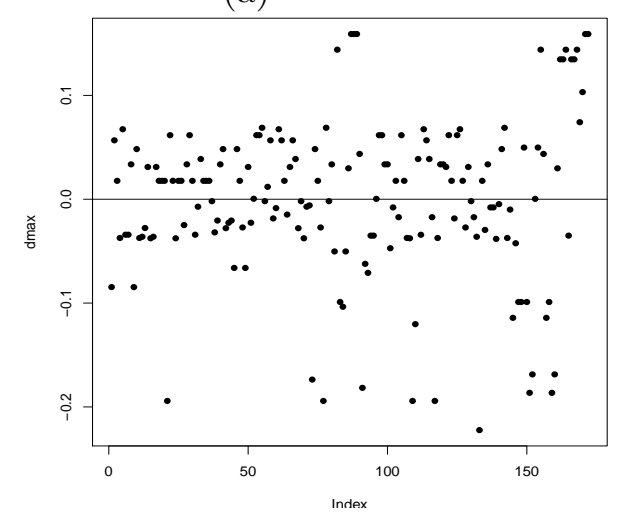

(b)

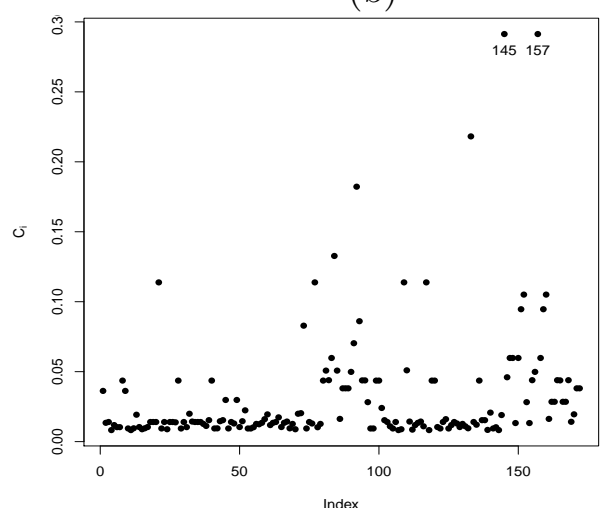

Figure 13: Index plots for $\boldsymbol{\theta}$ (case-weight perturbation): (a) $\mathbf{d}_{\max }$ and (b) total local influence.

\section{Response perturbation}

The influence of perturbing the observed response $Y$ will be analyzed. The value for the maximum curvature obtained is $C_{\mathbf{d}_{\text {max }}}=10.41$. Figure 14 display the index plots for $\mathbf{d}_{\max }$ and total local influence $C_{i}$. We may conclude that the observations 96 and 153 are possible influential points.

(a)

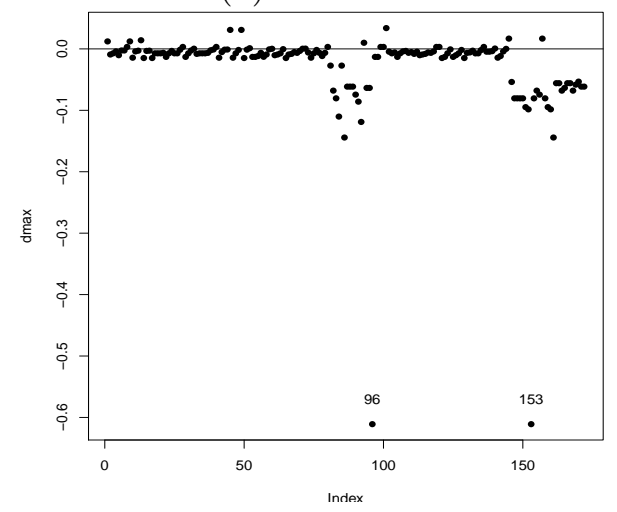

(b)

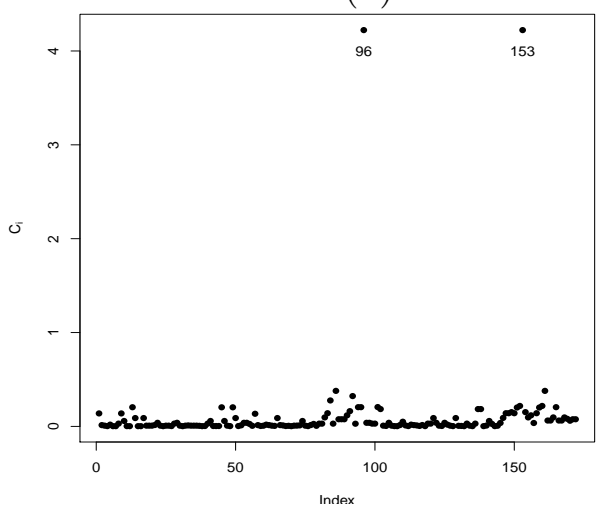

Figure 14: Index plots for $\boldsymbol{\theta}$ (response perturbation): (a) $\mathbf{d}_{\max }$ and (b) total local influence.

The global influential analysis indicates that the observations 92 and 133 are possible influential. The 92th observation has the large lifetime of the group 2 and the 133th observation has the smallest lifetime of the group 1. Under the local influential analysis (case-weight perturbation), the observations 145 and 157 are detected and they represent the smallest lifetimes of the group 2 with lifetimes $t_{145}=t_{157}=1$. Finally, with the local influential analysis (response perturbation), the detected observations 96 th and 153th are the intermediary measures of the group 2 . 


\section{ACCEPTED MANUSCRIPT}

\subsubsection{Residual analysis}

In order to detect possible outliers as well as departures from the assumptions made for the ESC regression model, we present in Figure 15 the normal probability plot with generated confidence band and the index plot for the martingale-type residual. By analyzing these plots, the asymmetry is observed. However, there is no indication of departures from the assumptions made for the model as well as the presence of outlying observations.

(a)

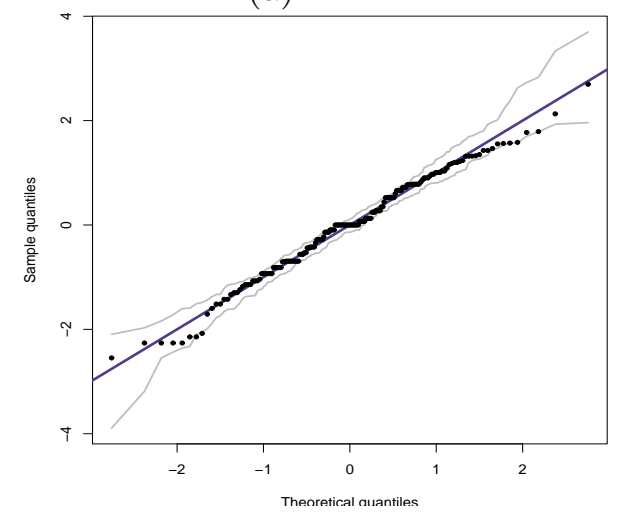

(b)

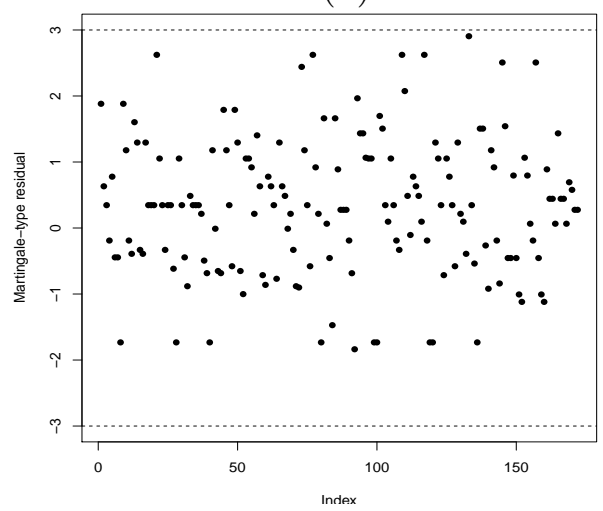

Figure 15: (a) Normal probability plot with envelope for the martingale-type residual $r_{D_{i}}$ from the fitted ESC regression model to the entomology data. (b) Index plot of the martingale-type residual $r_{D_{i}}$ for the entomology data.

Finally, in order to assess if the model is appropriate, the empirical and estimated survival functions of the ESC regression model are plotted in Figure 16 for the different groups. We may conclude from the plots that the ESC regression model provides a suitable fit to the entomology data.

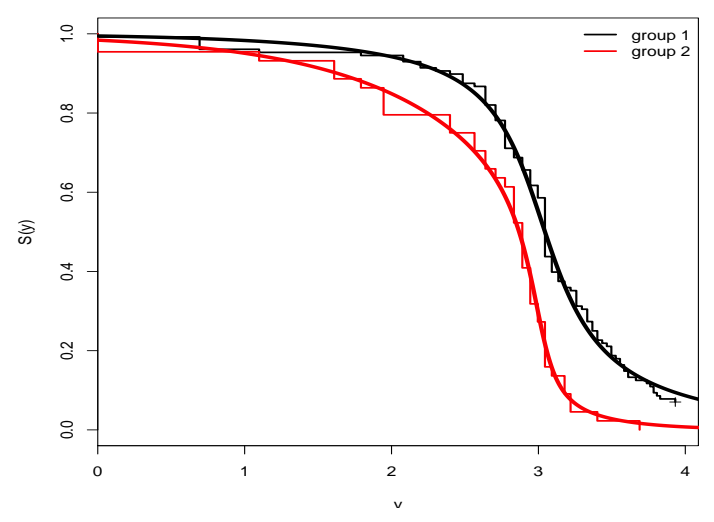

Figure 16: Estimated and empirical survival functions for the entomology data.

\section{Conclusions}

In this paper, we propose a general class of exponentiated sinh Cauchy (ESC) regression models, where the mean, dispersion, skewness and bimodal parameters vary across observations through re- 


\section{ACCEPTED MANUSCRIPT}

gression structures. The former class of regression models is very suitable for modeling censored and uncensored lifetime data. The proposed model serves as an important extension to several existing regression models and could be a valuable addition to the literature. We use the GAMLSS script in the $\mathrm{R}$ package to obtain the maximum likelihood estimates and perform asymptotic tests for the model parameters based on the asymptotic distribution of the estimates. We offer some interesting insights, especially regarding model checking, and provide applications of influence diagnostics (global, local and total influence) in the proposed class of regression models with censored data. We also discuss the adequacy of the regression models via martingale-type and quantile residuals. Several simulation studies are performed for different parameter settings, sample sizes and censoring percentages. Moreover, the usefulness of the model is also illustrated through the analysis of real data sets. Finally, the proposed algorithm for estimating the parameters in the probability density, cumulative distribution and quantile functions has been coded and implemented in the GAMLLS script available in the paper.

\section{Appendix: Script for the ESC regression model}

Here, we provide a brief discussion of the script for the ESC regression model implemented in the GAMLSS R package. The first step to run the codes is load the gamlss and gamlss.cens packages as well as the ESC model codes. After loading the codes, the pdf, cdf and qf will be available to be used. It is also available the function to generate random values having the ESC distribution.

In the example below, we present two ways to obtain the MLEs of the model parameters for uncensored and censored data. For both models, $\mathrm{m} 1$ and $\mathrm{m} 2$, we are modeling all parameters with the explanatory variable $X$. After fitting the selected models, we can access the goodness-of-fit statistics. Finally, the codes to access the residual analysis, for uncensored and censored, respectively, are reported.

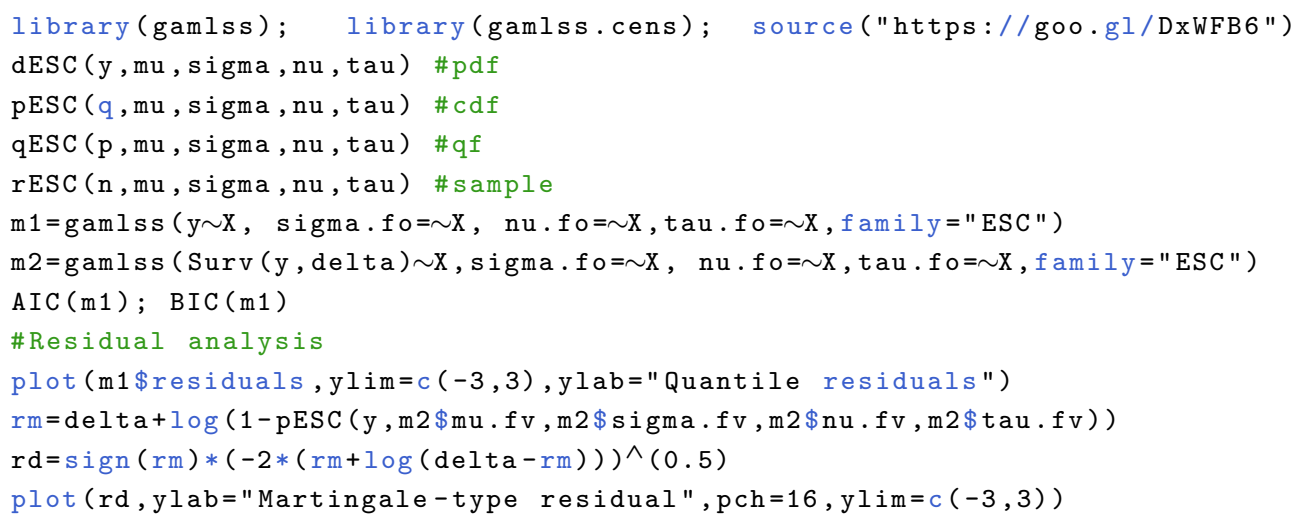

\section{References}

Atkinson, A.C. (1985). Plots, transformations, and regression: an introduction to graphical methods of diagnostic regression analysis. Oxford: Clarendon Press.

Barlow, W.E. and Prentice, R.L. (1988). Residuals for relative risk regression. Biometrika, 75, 65-74. 


\section{ACCEPTED MANUSCRIPT}

Cancho, V.G., Ortega, E.M.M. and Bolfarine, H. (2009). The log-exponentiated-Weibull regression models with cure rate: local influence and residual analysis. Journal of Data Science, 7, 433-458.

Cook, R.D. (1986). Assessment of local influence. Journal of the Royal Statistical Society, 48, $133-169$.

Cook, R.D. and Weisberg, S. (1982). Residuals and Influence in Regression. New York: Chapman and Hill.

Cooray, K. (2013). Exponentiated Sinh Cauchy Distribution with Applications. Communications in StatisticsTheory and Methods, 42, 3838-3852.

Cordeiro, G.M., Alizadeh, M., Ramires, T.G. and Ortega, E.M.M. (2016). The Generalized Odd Half-Cauchy Family of Distributions: Properties and Applications. Communications in Statistics-Theory and Methods. DOI:10.1080/03610926.2015.1109665.

Cordeiro, G.M., Ortega, E.M.M. and Ramires, T.G. (2015). A new generalized Weibull family of distributions: mathematical properties and applications. Journal of Statistical Distributions and Applications, 2, 1-25.

Dunn, P.K. and Smyth, G.K. (1996). Randomized quantile residuals. Journal of Computational and Graphical Statistics, 5, 236-244.

Fleming, T.R. and Harrington, D.P. (1991). Counting processes and survival analysis. John Wiley \& Sons.

Gradshteyn, I.S. and Ryzhik, I.M. (2007). Table of Integrals, Series, and Products, seventh edition. Academic Press, San Diego.

Gupta, R.C., Gupta, P.L. and Gupta, R.D. (1998). Modeling failure time data by Lehman alternatives. Communications in Statistics Theory and Methods, 27 , 887-904.

Gupta, R.D. and Kundu, D. (2001). Exponentiated exponential family: an alternative to Gamma and Weibull distributions. Biometrical Journal, 43 , 117-130.

Hashimoto, E.M., Ortega, E.M.M., Cordeiro, G.M. and Barreto, M.L. (2012). The Log-Burr XII Regression Model for Grouped Survival Data. Journal of biopharmaceutical statistics, 22, 141-159.

Leiva, V., Barros, M., Paula, G.A., Galea, M. (2007). Influence diagnostics in log-Birnbaum-Saunders regression models with Censored Data. Computational Statistics and Data Analysis, 51, 5694-5707.

Lesaffre, E. and Verbeke, G. (1998). Local influence in linear mixed models. Biometrics, 54, 570-582.

Ortega, E.M.M., Bolfarine, H. and Paula, G.A. (2003). Influence diagnostics in generalized log-gamma regression models. Computational Statistics and Data Analysis, 42, 165-186.

Ortega, E.M.M., Cordeiro, G.M., Lemonte, A.J. and da Cruz, J.N. (2016). The odd Birnbaum-Saunders regression model with applications to lifetime data. Journal of Statistical Theory and Practice, 10, $780-804$.

Ortega, E.M.M., Cordeiro, G.M., Campelo, A.K., Kattan, M.W. and Cancho, V.G. (2015). A power series beta Weibull regression model for predicting breast carcinoma. Statistics in medicine, 34, 1366-1388.

Ortega, E.M.M., Cordeiro, G.M., Hashimoto, E.M. and Cooray, K. (2014). A log-linear regression model for the odd Weibull distribution with censored data. Journal of Applied Statistics, 41, 1859-1880.

Ortega, E.M.M., Cordeiro, G.M. and Kattan, M.W. (2013). The log-beta Weibull regression model with application to predict recurrence of prostate cancer. Statistical Papers, 54, 113-132. 


\section{ACCEPTED MANUSCRIPT}

Ortega, E.M.M., Paula, G.A. and Bolfarine, H. (2008). Deviance residuals in generalized log-gamma regression models with censored observations. Journal of Statistical Computation and Simulation, 78, 747-764.

Pinheiro, A.P. (2008). Caracterização genética e biomètrica das populações de camarão rosa Farfantepenaeus brasiliensis de três localidades da costa do Rio Grande do Norte. Ecology and Natural Resources, Federal Univ. of São Carlos, SP, Brazil.

R Core team. (2015). R: A language and environment for statistical computing. R Foundation for Statistical Computing, Vienna, Austria. URL: https://www.R-project.org/.

Ramires, T.G., Ortega, E.M.M., Cordeiro, G.M. and Hens, N. (2016). A new bimodal flexible distribution for lifetime data. Journal of Statistical Computation and Simulation, 88, 2450-2470.

Rigby, R.A. and Stasinopoulos, D.M. (2005). Generalized additive models for location, scale and shape. Journal of the Royal Statistical Society: Series C (Applied Statistics), 54, 507-554.

Silva, M.A., Bezerra-Silva, G.C.D., Vendramim, J.D. and Mastrangelo, T. (2013). Sublethal effect of neem extract on Mediterranean fruit fly adults. Revista Brasileira de Fruticultura, 35, 93-101.

Silva, G.O., Ortega, E.M.M., Cancho, V.G. and Barreto, M.L. (2008). Log-Burr XII regression models with censored data. Computational Statistics and Data Analysis, 52, 3820-3842.

Stasinopoulos, D.M. and Rigby, R.A. (2007). Generalized additive models for location scale and shape (GAMLSS) in R. Journal of Statistical Software, 23, 1-46.

Therneau, T.M., Grambsch, P.M and Fleming, T.R. (1990). Martingale-based residuals for survival models. Biometrika, 77, 147-160.

Zhu, H., Ibrahim, J.G., Lee, S. and Zhang, H. (2007). Perturbation selection and influence measures in local influence analysis. The Annals of Statistics, 35, 2565-2588. 